\title{
Population and mass imbalance in atomic Fermi gases
}

\author{
J. E. Baarsma, ${ }^{*}$ K. B. Gubbels, and H. T. C. Stoof \\ Institute for Theoretical Physics, Utrecht University, Leuvenlaan 4, NL-3584 CE Utrecht, The Netherlands
}

(Received 21 December 2009; published 19 July 2010)

\begin{abstract}
We develop an accurate theory of resonantly interacting Fermi mixtures with both spin and mass imbalance. We consider Fermi mixtures with arbitrary mass imbalances but focus, in particular, on the experimentally available ${ }^{6} \mathrm{Li}^{40} \mathrm{~K}$ mixture. We determine the phase diagram of the mixture for different interactions strengths that lie on the BCS side of the Feshbach resonance. We also determine the universal phase diagram at unitarity. We find, for the mixtures with a sufficiently large mass imbalance, which includes the ${ }^{6} \mathrm{Li}^{-4}{ }^{40} \mathrm{~K}$ mixture, a Lifshitz point in the universal phase diagram that signals an instability toward a supersolid phase.
\end{abstract}

DOI: 10.1103/PhysRevA.82.013624

PACS number(s): 03.75.-b

\section{INTRODUCTION}

Ultracold quantum gases of fermionic atoms are at the center of attention of both experimental and theoretical physicists. Because of the amazing experimental control in these gases, they offer the possibility of experimentally exploring various pairing phenomena. As a result, many fundamental discoveries have already been made, for example, the realization of the crossover from a Bardeen-Cooper-Schrieffer (BCS) superfluid of loosely bound Cooper pairs to a Bose-Einstein condensate (BEC) of tightly bound molecules, also called the BEC-BCS crossover.

For high- $T_{c}$ superconductors, the size of a Cooper pair is comparable to the average distance between the electrons. This is analogous to the intermediate regime in the BEC-BCS crossover. In the BCS regime, the distance between two particles making up a Cooper pair is much larger than the average distance between particles, whereas in the BEC regime the distance between particles within a pair is much smaller than the average interparticle distance. If the size of a pair can be manipulated, it is possible to go smoothly from one regime to the other. This manipulation can be achieved in an atomic Fermi gas, where the interaction strength between the atoms in the two different spin states can be controlled with a Feshbach resonance. The smooth BEC-BCS crossover was eventually realized in a trapped gas of ${ }^{40} \mathrm{~K}$ [1] and ${ }^{6} \mathrm{Li}$ atoms [2-6].

For a Fermi mixture pairing is possible for an equal amount of particles in each spin state. However, pairing is absent for the noninteracting system with all particles in one spin state. Therefore, as a function of population imbalance, there must exist a phase transition at low temperatures. Experimentally, this transition was studied for the strongly interacting and mass-balanced case $[7,8]$, and the phase diagram was found to be governed by a tricritical point that resulted in the observation of phase separation [8,9]. Sarma superfluidity is also likely to be present in this system $[10,11]$ but has not been unambiguously identified yet.

Besides an imbalance in the particle densities, there can also be an imbalance between the masses of the particles. Consequences of these imbalances are interesting to study, also because imbalanced Fermi mixtures exist in certain condensed-matter systems in a magnetic field, in nuclear

\footnotetext{
*J.E.Baarsma@uu.nl
}

matter, and even in the quark-gluon plasma that is supposed to be present in the core of heavy neutron stars [12,13].

While studying mass-imbalanced Fermi mixtures, we first focused on the mixture with a mass ratio of 6.7 , corresponding to a ${ }^{6} \mathrm{Li}^{-40} \mathrm{~K}$ mixture. This resulted in a Letter [14] in which we presented the phase diagram of this mixture as a function of polarization and temperature in the strongly interacting limit, or the so-called unitarity limit, where the scattering length of the interparticle interaction diverges. In the unitarity limit, the size of the Cooper pairs is comparable to the average interparticle distance and the pairing is a many-body effect. As a result, the mass imbalance has profound effects on the pairing, because the imbalance strongly affects the two Fermi spheres that are present in the system. We showed that the phase diagram of the ${ }^{6} \mathrm{Li}^{-}{ }^{40} \mathrm{~K}$ mixture contains a Lifshitz point for a majority of heavy fermions [14]. Typically, Lifshitz points are found at weak interactions where the critical temperatures are very low. However, we found that in the strongly interacting limit the phase diagram of the ${ }^{6} \mathrm{Li}^{-}{ }^{40} \mathrm{~K}$ mixture already contains a Lifshitz point at accessible temperatures (see Fig. 17). At a Lifshitz point the phase transition undergoes a dramatic change of character. Rather than preferring a homogeneous order parameter, the system now forms an inhomogeneous superfluid.

The possibility of an inhomogeneous superfluid was investigated earlier by Larkin and Ovchinnikov (LO), who considered a superfluid with a single standing-wave order parameter [15], which is energetically more favorable than the plane-wave case studied by Fulde and Ferrell (FF) [16]. Since the LO phase results in periodic modulations of the particle densities, it is a supersolid [17]. The FF and LO phases have intrigued the condensed-matter community for many decades, but only very recently has strong evidence for the FFLO phase been obtained in a one-dimensional imbalanced Fermi mixture of two spin states [18]. Theoretically, it is challenging to describe the phase diagram below a Lifshitz point. The most stable states are likely to be complicated superpositions of standing waves, where different ansatzes lead to different stability regions $[19,20]$.

Initially, our focus was on the ${ }^{6} \mathrm{Li}^{4}{ }^{40} \mathrm{~K}$ mixture, because this is experimentally a very promising mixture, where several accessible Feshbach resonances are identified [21] and both species have also been simultaneously cooled into the degenerate regime [22]. In contrast to our previous work, we now consider Fermi mixtures with an arbitrary mass ratio. Moreover, apart from the unitarity limit, we also cover the 
$\mathrm{BCS}$ regime. We do not calculate phase diagrams on the BEC side of the Feshbach resonance, because in that regime we should include thermal molecules in our calculations, which requires a different theory. Due to the population and the mass imbalance, the two Fermi spheres in the system are typically mismatched, which can induce phase separation, with the location of the tricritical point depending on the mass ratio and the interaction strength [23]. In this paper, we also consider Lifshitz instabilities and find a multicritical point for the mass-balanced case at a very weak interaction, as shown in Fig. 13. At this interaction strength the Lifshitz point and the tricritical point occur for the same polarization and temperature. Furthermore, in the imbalanced Fermi mixture the quasiparticle dispersions in the superfluid phase also give rise to gapless Sarma superfluidity. For the mass-balanced Fermi mixture and for the ${ }^{6} \mathrm{Li}^{40} \mathrm{~K}$ mixture we therefore also calculated the regions in the phase diagrams where the superfluid is gapless.

In our Letter [14], we showed that although meanfield theory vastly overestimates critical temperatures in the unitarity limit, it is very useful for a qualitative description of the physics. From the mass-balanced case, we know that the critical temperatures found using mean-field techniques are lowered mainly by two effects, namely, the fermionic self-energies and the screening of the interaction due to particle-hole fluctuations [24,25]. After discussing mean-field theory for the imbalanced Fermi gases, we take both these effects into account in the unitarity limit for the mass-balanced case and for the experimentally interesting case of the ${ }^{6} \mathrm{Li}-$ ${ }^{40} \mathrm{~K}$ mixture. This leads to results that compare well with Monte Carlo calculations [26] and, for equal masses, also with experiment [9]. Our procedure gives a reduction in the mean-field critical temperatures by a factor of 3 . This makes it experimentally more difficult, but not impossible, to reach the superfluid regime also for the mass-imbalanced case. Very importantly, the Lifshitz point remains present in the phase diagram after taking fluctuations into account. This hopefully brings the observation of inhomogeneous superfluidity within experimental reach.

This paper is organized as follows. We start by discussing the interactions in Fermi mixtures in Sec. II. In Sec. III, we give a brief discussion of the Landau theory that we use to describe phase transitions. In particular, we introduce the Landau thermodynamic potential and the order parameter. Next, in Sec. IV, we discuss the mean-field theory that we use to calculate phase diagrams, which are presented in Sec. IV C. We discuss the phase diagrams for three mass ratios at different interaction strengths, to explore the various topologies of the phase diagrams that can arise. After that, we discuss in more detail the effects of the mass imbalance. We then also explore the effect of the interaction strength on the position of the tricritical points and Lifshitz points in the phase diagram. Subsequently, we discuss the presence of the superfluid Sarma phase for the mass-balanced case and the ${ }^{6} \mathrm{Li}^{4}{ }^{40} \mathrm{~K}$ mixture, both at unitarity. All these calculations use, in the first instance, mean-field theory. Then, in Sec. V, we include fluctuation effects to obtain more quantitative results for the mass-balanced case and the ${ }^{6} \mathrm{Li}^{40} \mathrm{~K}$ mixture. We focus here on the unitarity limit, although fluctuation effects could also be easily incorporated in the BCS limit of the Feshbach resonance. Finally, more calculations are given in the appendixes. In Appendix A it is explained how the thermodynamic potential for the mass-imbalanced Fermi gas is obtained, while Appendix B contains the calculations for the amplitudes of relevant Feynman diagrams.

\section{INTERACTIONS AND FESHBACH RESONANCES}

In this paper we study phase transitions in an imbalanced Fermi gas at different interaction strengths. This is relevant, in particular, if the interaction strength is experimentally under control. In atomic Fermi mixtures the interspecies interaction can be controlled using a Feshbach resonance [27]. The effect of the microscopic interaction potential can be studied via the two-body transition operator $\hat{T}^{2 \mathrm{~B}}$. The matrix elements of this transition operator are directly related to the scattering amplitudes. It is defined by

$$
\hat{V}\left|\psi_{\mathbf{k}}^{(+)}\right\rangle \equiv \hat{T}^{2 \mathrm{~B}}|\mathbf{k}\rangle,
$$

where $\left|\psi_{\mathbf{k}}^{(+)}\right\rangle$are the scattering states and $\hat{V}$ is the microscopic interaction potential. To find an expression for $\hat{T}^{2 \mathrm{~B}}$ we start with the Lippmann-Schwinger equation,

$$
\hat{T}^{2 \mathrm{~B}}=\hat{V}+\hat{V} \frac{1}{z-\hat{H}_{0}} \hat{T}^{2 \mathrm{~B}},
$$

where $z=E+i 0$ and the notation $i 0$ implies the limit $i \varepsilon$ with $\varepsilon \downarrow 0$. In this paper we study an imbalanced Fermi gas with a point interaction

$$
V\left(\mathbf{x}-\mathbf{x}^{\prime}\right) \simeq V_{0} \delta\left(\mathbf{x}-\mathbf{x}^{\prime}\right),
$$

where $V_{0}$ is negative, since we are interested in an attractive interaction. If we now consider the Lippmann-Schwinger equation at zero energy, $z=0$, multiply both sides with $\left\langle\mathbf{k}^{\prime}\right|$ from the left and with $\left|\mathbf{k}^{\prime \prime}\right\rangle$ from the right, and insert a completeness relation in the second term on the right-hand side, we obtain

$$
\frac{1}{T^{2 \mathrm{~B}}(0)}=\frac{1}{V_{0}}+\int \frac{d \mathbf{k}}{(2 \pi)^{3}} \frac{1}{2 \varepsilon(\mathbf{k})},
$$

where half the reduced kinetic energy, $\varepsilon(\mathbf{k})=\hbar^{2} \mathbf{k}^{2} / 2 m$, is the kinetic energy associated with a mass $m$ that is equal to twice the reduced mass, namely,

$$
\frac{2}{m}=\frac{1}{m_{+}}+\frac{1}{m_{-}} .
$$

Here, $m_{+}$and $m_{-}$are the masses of a light and a heavy particle, respectively.

The two-body transition matrix is related to the $s$-wave scattering length $a$ by

$$
\frac{1}{T^{2 \mathrm{~B}}(0)}=\frac{m}{4 \pi \hbar^{2} a} .
$$

Since we are interested in the behavior of the Fermi gas at ultralow temperatures, we can use a cutoff momentum $\hbar \Lambda$ to evaluate the integral in Eq. (4) and we obtain, using Eq. (6), a relation between the scattering length $a$ and the microscopic interaction potential $V_{0}$, namely,

$$
a=\frac{m \pi V_{0}}{2 m \Lambda V_{0}+4 \pi^{2} \hbar^{2}} .
$$




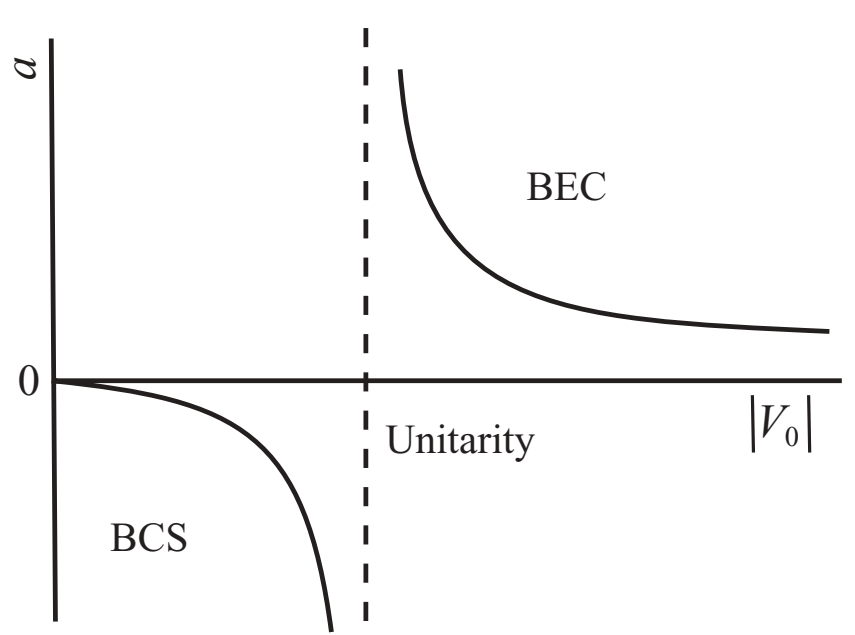

FIG. 1. The $s$-wave scattering length $a$ as a function of the microscopic interaction strength $V_{0}$. Here $V_{0}$ is negative, since we are considering an attractive interaction.

This relation is shown in Fig. 1. It can be seen that for small values of $V_{0}$ the scattering length is negative, which means that the Fermi mixture is in the BCS regime. Then, for $V_{0}=$ $-(2 \pi \hbar)^{2} / m \Lambda$, the scattering length diverges; this is called the unitarity limit. For large values of $V_{0}$ the scattering length is positive and the Fermi mixture is in the BEC regime.

For $a>0$ the microscopic interaction has a bound state with an energy $E=-\hbar^{2} / m a^{2}$. This is the single-channel picture of a Feshbach resonance. In this paper we focus on the unitarity limit and on the BCS side of the resonance. Thus, we look at the case where $a$ diverges and at negative scattering lengths $a$. For those scattering lengths we can use the single-channel picture, as long as the Feshbach resonance is sufficiently broad. Namely, in the limit of a broad resonance the amplitude to be in the bare molecular state of the Feshbach resonance turns out to be very low $[6,28]$.

\section{LANDAU THEORY OF PHASE TRANSITIONS}

To study the critical behavior of a system, in our case an imbalanced Fermi gas, we consider the Landau thermodynamic potential density $\omega_{\mathrm{L}}[\Delta(\mathbf{x})]$, with $\Delta(\mathbf{x})$ the superfluid order parameter. Near the phase transition, where the BCS order parameter $\Delta(\mathbf{x})$ is small, the Landau thermodynamic potential density can be expanded as $[29,30]$

$$
\omega_{\mathrm{L}}\left(\Delta ; \mu_{\sigma}, T\right)=\gamma|\nabla \Delta|^{2}+\alpha|\Delta|^{2}+\frac{\beta}{2}|\Delta|^{4}+\ldots,
$$

where the dots denote the higher orders in $|\Delta|^{2}$ and in gradients $|\nabla \Delta|^{2}$. The Landau coefficients in the thermodynamic potential all depend on the temperature and on the chemical potentials of the two fermion species. If, in the thermodynamic potential, all coefficients are positive, the minimum of the thermodynamic potential is located at $\langle\Delta(\mathbf{x})\rangle$ equal to zero and the system will be in the normal state. In contrast, a phase transition to a superfluid state has occurred when the position of the global minimum is located at a nonzero order parameter $\langle\Delta(\mathbf{x})\rangle$, which describes a condensate of bosonic pairs. In the case where $\gamma$ is positive, it costs energy to have a spatially varying superfluid. It is then energetically favorable for the system to be homogeneous, and therefore we can restrict ourselves to a pairing field $\Delta$ independent of position.

We consider first the case where $\gamma$ is positive and the system is homogeneous. For high temperatures all coefficients in the thermodynamic potential will be positive and the system will be in the normal state. But for low temperatures it can occur that certain coefficients change sign. Suppose that in the Landau thermodynamic potential $\alpha$ is negative and all other coefficients are positive. The minimum of the thermodynamic potential will then be attained at some nonzero $\langle\Delta\rangle$ and the Fermi gas will be in the superfluid state. Thus, as $\alpha$ changes sign a phase transition takes place. The temperature at which the transition from the normal state to the superfluid state occurs, for given chemical potentials, can be determined by equating the quadratic coefficient to zero, $\alpha\left(T_{\mathrm{c}}\right)=0$, where $T_{\mathrm{c}}$ is called the critical temperature. The phase transition just described is called a second-order phase transition and it is characterized by the fact that the minimum of the thermodynamic potential shifts away from zero continuously [see Fig. 2(a)].

It is also possible to have a first-order phase transition. To explain a first-order phase transition we consider the situation where, in the thermodynamic potential density, all coefficients but the fourth-order coefficient $\beta$ are positive. The thermodynamic potential will then typically have two minima. One of these is located at $\Delta$ equal to zero and the other one will be located at a nonzero value of the order parameter. For higher temperatures the minimum located at zero is a global minimum and the Fermi gas is in the normal state. If the temperature is lowered there will be a point where the two minima are equal, and for even lower temperatures the minimum located at a nonzero order parameter $\langle\Delta\rangle$ is the global minimum. The system is then in the superfluid state. The first-order phase transition takes place when these two minima are equal, that is, when $\omega_{\mathrm{L}}(0)=\omega_{\mathrm{L}}(\langle\Delta\rangle)$. In contrast to a second-order phase transition, the location of the global minimum of the thermodynamic potential now changes discontinuously from being zero to a nonzero $\langle\Delta\rangle$ [see Fig. 2(b)].

Next, we consider the case where $\gamma$ is negative. The system can then gain energy when the order parameter varies in space. Thus, instead of being constant, the order parameter will now depend on position. FF studied the plane-wave solution [16]

$$
\Delta(\mathbf{x})=\Delta_{0} e^{i \mathbf{k} \cdot \mathbf{x}},
$$

Second-order phase transition First-order phase transition
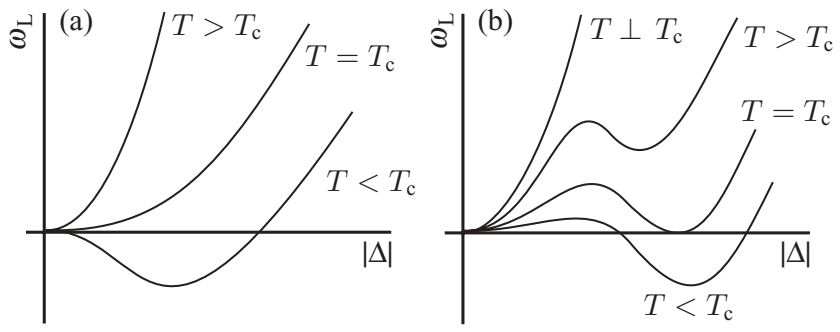

FIG. 2. The thermodynamic potential density $\omega_{\mathrm{L}}(|\Delta|)$ as a function of the order parameter $\Delta$. The behavior of $\omega_{\mathrm{L}}(|\Delta|)$ for different temperatures (a) when a second-order phase transition occurs and (b) when a first-order phase transition occurs. 
while LO considered a superfluid with the single standingwave order parameter [15]

$$
\Delta(\mathbf{x})=\Delta_{0} \cos (\mathbf{k} \cdot \mathbf{x}),
$$

which turns out to be energetically more favorable than the plane-wave case. Superpositions of more than two plane waves are also possible.

In the LO phase the wave function of the bosonic pairs is periodic and therefore there will also exist a periodicity in the atomic density. This periodic structure shows itself in the diagonal elements of the one-particle density matrix $n\left(\mathbf{x}, \mathbf{x}^{\prime}\right)$ and is therefore called diagonal long-range order. This diagonal long-range order is what characterizes a solid. In a fermionic superfluid a fraction of the Cooper pairs is in the lowest energy eigenstate. There thus exists a long-range order between the positions of the pairs. It is also said that the two-particle density matrix $g\left(\mathbf{x}, \mathbf{x}^{\prime}\right)$ has off-diagonal long-range order, which implies that $g\left(\mathbf{x}, \mathbf{x}^{\prime}\right)$ does not vanish in the limit $\left|\mathbf{x}-\mathbf{x}^{\prime}\right| \rightarrow \infty$ and characterizes a superfluid. In the case where $\gamma$ is negative a transition occurs from the normal state to a superfluid where the atomic density in the superfluid has a periodic structure. Then there exists both diagonal long-range order as well as off-diagonal long-range order and the state is both solid and superfluid. This is called a supersolid phase [17,30].

Further on, we show that in a mass-imbalanced Fermi gas, not only a second-order phase transition can occur, but also a first-order transition and even a transition to an inhomogeneous superfluid, depending on the values of the two chemical potentials. Therefore, there must be points in the phase diagram where the character of the phase transition changes. If the phase transition changes from being second order to first order, there will be a tricritical point [31]. This point can thus be found by setting

$$
\alpha\left(T_{\mathrm{c} 3}\right)=\beta\left(T_{\mathrm{c} 3}\right)=0,
$$

where $T_{\mathrm{c} 3}$ is the tricritical temperature. For temperatures higher than the tricritical temperature the phase transition from the normal to the superfluid state is of second order, while for $T<T_{\mathrm{c} 3}$ there is a first-order phase transition and phase separation occurs.

The phase transition could also change from being a transition from the normal state to a homogeneous superfluid to a transition from the normal state to a supersolid. The point in the phase diagram where this occurs is called a Lifshitz point [31]. This point can be computed by demanding

$$
\alpha\left(T_{L}\right)=\gamma\left(T_{L}\right)=0,
$$

where $T_{L}$ is called the Lifshitz temperature. For temperatures lower than the Lifshitz temperature the transition will be from a normal state to a superfluid state where the bosonic pairs have a nonzero momentum. Superfluidity at nonzero momentum can be established in many ways, and due to this variety of possibilities it is difficult to predict which kind of superfluidity will be present below the Lifshitz point. However, they all have to emerge from the Lifshitz point and therefore it is important to know the position of the Lifshitz point.

Apart from the preceding two possibilities, we can think of other scenarios for the change in character of the phase transitions. But in the phase diagrams we calculated for the imbalanced Fermi gas, we only found tricritical points and Lifshitz points. Therefore, these are the only two possibilities we discuss in the following.

\section{MEAN-FIELD THEORY}

In this section we present the mean-field thermodynamic potential, which is an approximation to the exact Landau thermodynamic potential for the Fermi gas with population and mass imbalance. If we have an expression for the Landau thermodynamic potential, we are able to determine the phase diagram.

Although mean-field theory does not contain all interactions present in a Fermi mixture, it turns out that mean-field theory already incorporates all the relevant physics determining the topology of the phase diagrams. Adding fluctuation effects only changes the phase diagrams quantitatively [14,24,25,32]. Because of the rather straightforward and transparent calculations in mean-field theory, we first discuss mean-field theory in some detail. Later we take fluctuation effects into account to obtain more quantitative results that we can compare with Monte Carlo calculations and for the mass-balanced case with experiment.

\section{A. Thermodynamic potential}

We consider a two-component Fermi mixture, that is, a mixture containing either a single fermionic species, for which two different hyperfine states are present, or consisting of two different fermionic species with access to a single hyperfine state. A balanced Fermi gas consists of a single species with an equal population of both spin states. In an imbalanced Fermi gas we allow the populations and masses to be different. To take into account a population imbalance we use different chemical potentials for the two (pseudo)spin states, while a mass imbalance implies that the particles in the two hyperfine states have different masses. The chemical potential and the mass of the fermions in state $|\sigma\rangle$ are denoted $\mu_{\sigma}$ and $m_{\sigma}$, respectively. From now on heavy particles are always denoted with a minus sign and light particles with a plus sign, thus $\sigma= \pm$.

The mean-field thermodynamic potential for the imbalanced Fermi gas is given by

$$
\begin{aligned}
\omega_{\mathrm{L}}(|\Delta|)= & -\frac{|\Delta|^{2}}{T^{2 \mathrm{~B}}(0)}+\int \frac{d \mathbf{k}}{(2 \pi)^{3}}\{\varepsilon(\mathbf{k})-\mu-\hbar \omega(\mathbf{k}) \\
& \left.+\frac{|\Delta|^{2}}{2 \varepsilon(\mathbf{k})}-\frac{1}{\beta} \sum_{\sigma} \log \left[1+e^{-\beta \hbar \omega_{\sigma}(\mathbf{k})}\right]\right\}
\end{aligned}
$$

where the two-body transition matrix $T^{2 \mathrm{~B}}(0)$ is given by Eq. (6). This thermodynamic potential is a direct generalization of the thermodynamic potential for a balanced Fermi gas [30,33]. In expression (13), $\beta=1 / k_{\mathrm{B}} T$ is the inverse thermal energy, $\mu=\left(\mu_{+}+\mu_{-}\right) / 2$ is the average chemical potential, and half the reduced kinetic energy is $\varepsilon(\mathbf{k})=$ $\left[\varepsilon_{+}(\mathbf{k})+\varepsilon_{-}(\mathbf{k})\right] / 2$, with

$$
\varepsilon_{\sigma}(\mathbf{k})=\frac{\hbar^{2} \mathbf{k}^{2}}{2 m_{\sigma}} .
$$


The first terms in the thermodynamic potential (13) represent the BCS ground state of the mixture, where $\hbar \omega(\mathbf{k})$ is the average dispersion of the quasiparticles,

$$
\hbar \omega(\mathbf{k})=\sqrt{[\varepsilon(\mathbf{k})-\mu]^{2}+|\Delta|^{2}},
$$

with $|\Delta|$ the so-called BCS gap parameter. The complex pairing field $\Delta$ is, on average, related to the expectation value of the pair annihilation operator through

$$
\langle\Delta(\mathbf{x})\rangle=V_{0}\left\langle\hat{\psi}_{-}(\mathbf{x}) \hat{\psi}_{+}(\mathbf{x})\right\rangle,
$$

with $\hat{\psi}_{\sigma}(\mathbf{x})$ the fermionic annihilation operators.

The second part of the thermodynamic potential (13), namely, the part containing the logarithms, corresponds to the contribution of an ideal gas of quasiparticles. Here, $\hbar \omega_{\sigma}(\mathbf{k})$ is the dispersion relation of the quasiparticles in state $|\sigma\rangle$, given by

$$
\hbar \omega_{\sigma}(\mathbf{k})=\hbar \omega(\mathbf{k})-\sigma\left[2 h-\varepsilon_{+}(\mathbf{k})+\varepsilon_{-}(\mathbf{k})\right] / 2,
$$

with $h=\left(\mu_{+}-\mu_{-}\right) / 2$ half the difference in chemical potentials. For the unpolarized Fermi gas with equal masses the dispersions $\hbar \omega_{\sigma}(\mathbf{k})$ reduce to the average dispersion in Eq. (15). This dispersion is plotted in Fig. 3 for both the normal state [Fig. 3(a)] and the superfluid state [Fig. 3(b)]. In this case the superfluid is gapped and balanced. For $k=|\mathbf{k}|<\sqrt{2 m \mu} / \hbar$ the quasiparticle dispersion describes holelike excitations. If we mirror this holelike part of the quasiparticle dispersion, we obtain the negative dispersion of the particlelike states. For $k>\sqrt{2 m \mu} / \hbar$ the quasiparticle dispersion already describes the particlelike excitations.

In the case of a polarized superfluid with equal masses the dispersions $\hbar \omega_{\sigma}(\mathbf{k})$ in Fig. 3 are shifted by the difference in chemical potentials $2 h$. For $h>|\Delta|$ the dispersion of the majority species becomes negative. When this occurs, the occupation of the single-particle states associated with the negative part of the quasiparticle excitation branch actually lowers the ground-state energy. Since this leads to additional majority quasiparticles and, therefore, additional majority particles and minority holes in the ground state, the ground state becomes a polarized superfluid. The resulting gapless and polarized superfluid is called the Sarma phase [34]. For a Fermi gas with both mass and population imbalance the dispersions are
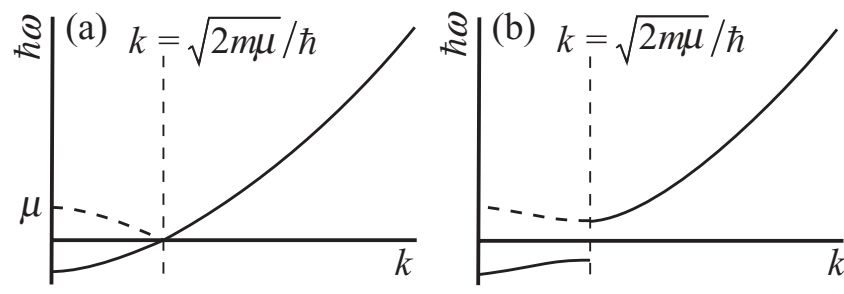

FIG. 3. Single-particle dispersions $\hbar \omega_{\sigma}(\mathbf{k})$ for the balanced Fermi gas. (a) Dispersions for $\Delta=0$ where the system is in the normal state. (b) The case where the system is in the superfluid state and, thus, the dispersions for nonzero $\Delta$. In both plots, dashed lines are the dispersions of the holelike excitations, while solid lines show the particlelike dispersions. The latter show more clearly the opening of a gap $2 \Delta$ at the Fermi level due to the formation of a condensate of Cooper pairs.
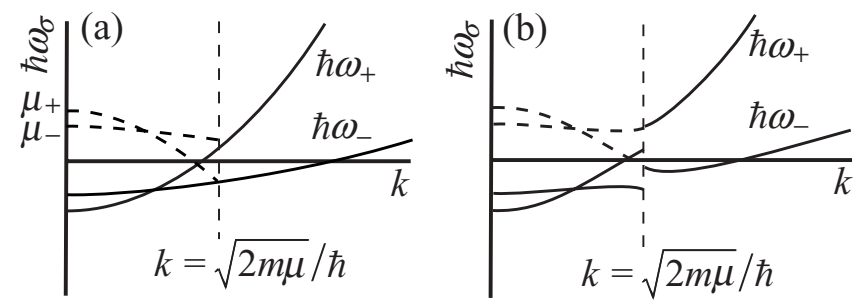

FIG. 4. Dispersions $\hbar \omega_{\sigma}(\mathbf{k})$ for the Fermi gas with both population and mass imbalance. (a) Dispersions for $\Delta=0$ where the system is in the normal state. (b) The case where the system is in the superfluid state and, thus, the dispersions for nonzero $\Delta$. The meaning of dashed and solid lines is the same as in Fig. 3.

depicted in Fig. 4, where Fig. 4(a) shows the dispersions for $\Delta=0$, and Fig. 4(b) for nonzero $\Delta$. The shape of the dispersions is changed due to the difference in mass. It can be seen that in this case also one of the dispersions, namely, $\hbar \omega_{-}(\mathbf{k})$, is negative so that it would correspond to a gapless Sarma superfluid.

\section{B. Landau coefficients}

For the imbalanced Fermi mixture, we want to study the phase transition from the normal state to the superfluid state. For that transition we want to obtain a phase diagram with the critical temperature as a function of the polarization, $P=\left(n_{+}-n_{-}\right) /\left(n_{+}+n_{-}\right)$, with $n_{\sigma}$ the density of particles in state $|\sigma\rangle$.

The particle densities $n_{\sigma}$ can be determined from the thermodynamic potential using [30]

$$
n_{\sigma}=-\left.\frac{\partial \omega_{\mathrm{L}}(\Delta)}{\partial \mu_{\sigma}}\right|_{\Delta=\langle\Delta\rangle} .
$$

Moreover, in Sec. III the critical conditions for the transition were explained. In mean-field theory, the quadratic Landau coefficient $\alpha$ is explicitly given by

$$
\begin{aligned}
\alpha & =\left.\frac{\partial \omega_{\mathrm{L}}}{\partial|\Delta|^{2}}\right|_{|\Delta|^{2}=0} \\
& =-\frac{1}{T^{2 \mathrm{~B}}(0)}+\int \frac{d \mathbf{k}}{(2 \pi)^{3}}\left\{\frac{1}{2 \varepsilon(\mathbf{k})}+\frac{N_{+}(\mathbf{k})+N_{-}(\mathbf{k})-1}{2[\varepsilon(\mathbf{k})-\mu]}\right\},
\end{aligned}
$$

where $N_{\sigma}(\mathbf{k})=1 /\left(\exp \left\{\beta\left[\varepsilon_{\sigma}(\mathbf{k})-\mu_{\sigma}\right]\right\}+1\right)$ are the Fermi distribution functions. To determine the temperature of the tricritical point, we also need to know the fourth-order Landau coefficient $\beta$. It is given by

$$
\begin{aligned}
\beta= & \left.\frac{\partial^{2} \omega_{\mathrm{L}}}{\left(\partial|\Delta|^{2}\right)^{2}}\right|_{|\Delta|^{2}=0} \\
= & \int \frac{d^{3} \mathbf{k}}{(2 \pi)^{3}} \frac{1}{4[\varepsilon(\mathbf{k})-\mu]^{2}}\left[\beta N_{+}(\mathbf{k})\left[N_{+}(\mathbf{k})-1\right]+\beta N_{-}(\mathbf{k})\right. \\
& \left.\times\left[N_{-}(\mathbf{k})-1\right]+\frac{1}{\varepsilon(\mathbf{k})-\mu}\left[1-N_{+}(\mathbf{k})-N_{-}(\mathbf{k})\right]\right] .
\end{aligned}
$$




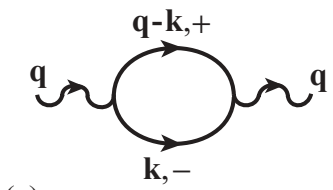

(a)

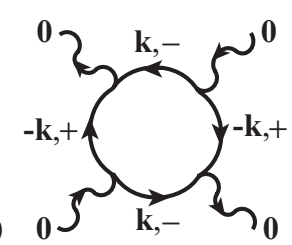

FIG. 5. (a) Ladder diagram with external momentum q. Wiggly lines denote Cooper pairs, which can break up into two fermions of different spin, denoted by normal lines. Here, $\mathbf{q}$ is the wave vector of the Cooper pairs, while $\mathbf{k}$ and $\mathbf{q}-\mathbf{k}$ are the wave vectors of the fermions. When $\mathbf{q}=\mathbf{0}$, the amplitude of this diagram corresponds to the quadratic Landau coefficient $\alpha$ in the thermodynamic potential. (b) Feynman diagram corresponding to the fourth-order Landau coefficient $\beta$.

Determining $\gamma$ from the mean-field thermodynamic potential is not possible, since we have assumed the bosonic pairing field $\Delta(\mathbf{x})$ to be independent of position. Nevertheless, there is a rather simple way to determine this coefficient, using Feynman diagrams [35]. The other coefficients could also have been determined using a diagrammatic language. Namely, the quadratic coefficient $\alpha$ corresponds to the so-called ladder diagram where the incoming and outgoing bosonic fields $\Delta$ have zero momentum [see Fig. 5(a)]. Physically, $\alpha$ can be interpreted as being proportional to the chemical potential of the Cooper pairs. The fourth-order coefficient $\beta$ has a diagrammatic representation with four external bosonic fields with zero momentum [see Fig. 5(b)].

For the transition to the supersolid phase, we consider the ladder diagram where the bosonic fields carry nonzero momentum $\mathbf{q}$, since the supersolid phase consists of bosonic pairs with nonzero momentum. The expression for this ladder diagram is

$$
\begin{aligned}
\alpha(\mathbf{q})= & -\frac{1}{T^{2 \mathrm{~B}}(0)}+\int \frac{d^{3} \mathbf{k}}{(2 \pi)^{3}} \\
& \times\left\{\frac{1}{2 \varepsilon(\mathbf{k})}+\frac{N_{+}(\mathbf{q}-\mathbf{k})+N_{-}(\mathbf{k})-1}{\varepsilon_{+}(\mathbf{q}-\mathbf{k})+\varepsilon_{-}(\mathbf{k})-2 \mu}\right\} .
\end{aligned}
$$

The actual shape of $\alpha(\mathbf{q})$ as a function of the external momentum $q$ depends on the values of the different parameters in this expression, such as the chemical potentials and the temperature (Fig. 6). Depending on those quantities the minimum of $\alpha(\mathbf{q})$ is attained either for zero or for nonzero external momentum. As explained in Sec. III, a second-order phase transition can occur, when a quadratic coefficient of the Landau theory changes sign. These coefficients are now given by $\alpha(\mathbf{q})$, where $\mathbf{q}$ is the wave vector of the bosonic pairs. The sign change occurs first for the minimum of $\alpha(\mathbf{q})$, which therefore determines whether or not the transition happens at nonzero q. The expression for the ladder diagram with nonzero external momentum can be expanded in even powers of $\mathbf{q}$,

$$
\alpha(\mathbf{q})=a_{0}+a_{1} \mathbf{q}^{2}+a_{2} \mathbf{q}^{4}+\ldots,
$$

where the dots denote higher-order powers in $\mathbf{q}^{2}$. If all the coefficients $a_{i}$ are positive, $\alpha(\mathbf{q})$ has a minimum for external momentum zero, so that a transition to the homogeneous superfluid phase occurs. But if $a_{1}$ is negative, $\alpha(\mathbf{q})$ has a minimum for a nonzero external momentum and therefore it first becomes zero at some nonzero value of $\mathbf{q}$. The minimum of the thermodynamic potential will then be located at a nonzero order parameter with a nonzero momentum. In other words, it will be energetically favorable for the bosonic pairs to have kinetic energy and the phase transition that occurs is a transition from the normal state to an inhomogeneous superfluid. Comparing this with the Landau theory in Sec. III, we see that in the expansion of $\alpha(\mathbf{q}), a_{0}$ can be identified with the quadratic coefficient $\alpha$ and $a_{1}$ can be identified with $\gamma$. Thus, from the ladder diagram with external momentum, an expression for $\gamma$ can be found, namely,

$$
\gamma=\left.\frac{\partial \alpha(\mathbf{q})}{\partial \mathbf{q}^{2}}\right|_{\mathbf{q}=\mathbf{0}} .
$$

Physically, $\gamma$ can be interpreted as being proportional to the inverse of the effective mass of the Cooper pairs.

\section{Results}

In this section the results using mean-field theory are presented. First, we present phase diagrams for three Fermi gases with different mass imbalances. Then we study the effect of the mass imbalance on the critical temperature for an unpolarized Fermi gas. After this, we study the effect of the interaction strength on the temperature corresponding to a tricritical point or a Lifshitz point. Finally, we also consider the superfluid Sarma phase.
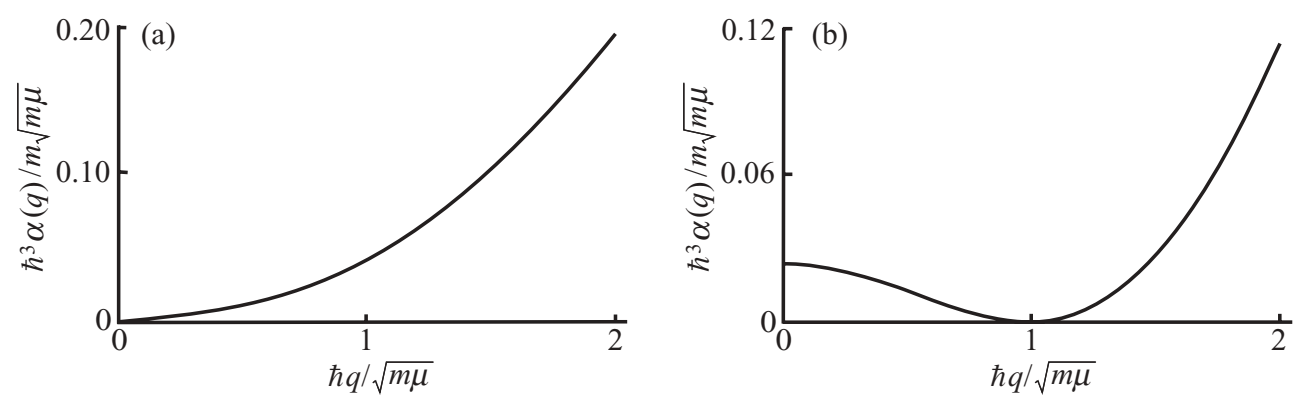

FIG. 6. Depending on the temperature and the chemical potentials, the minimum of $\alpha(\mathbf{q})$ is either at zero or at nonzero external momentum $\hbar q$. In both plots the mass ratio is $r=6.7$. (a) The minimum of $\alpha(\mathbf{q})$ is located at zero external momentum. The corresponding polarization is $P=-0.61$ and the temperature is $T / T_{F}=0.21$, with $T_{F}$ the Fermi temperature. (b) The minimum of $\alpha(\mathbf{q})$ is attained at a nonzero momentum. Here the polarization is $P=-0.7$ and the temperature is $T / T_{F}=0.13$. 


\section{Phase diagrams}

With the expressions for the Landau coefficients, the phase diagram can be calculated for a fixed mass ratio $r$ and a fixed interaction strength $1 / k_{\mathrm{F}} a$. We determine the phase diagram as a function of temperature $T$ and polarization $P=\left(n_{+}-n_{-}\right) /$ $\left(n_{+}+n_{-}\right)$. The mass ratio $r$ is given by $r=m_{-} / m_{+}$. The interaction strength is characterized by the $s$-wave scattering length $a$ and the Fermi momentum $k_{F}$, which is defined as

$$
k_{F}=\left(3 \pi^{2} n\right)^{1 / 3},
$$

where $n=n_{+}+n_{-}$is the total particle density. To obtain a phase diagram independent of the total particle density $n$, we scale the temperature with the reduced Fermi temperature,

$$
k_{B} T_{F}=\varepsilon_{F}=\frac{\hbar^{2} k_{F}^{2}}{2 m},
$$

where $m$ is twice the reduced mass, introduced in Eq. (5), and $k_{B}$ is Boltzmann's constant.

We present the phase diagram for three different mass ratios, namely, for $r=1$, which is the mass-balanced case, $r=6.7$, which corresponds to a ${ }^{6} \mathrm{Li}^{-}{ }^{40} \mathrm{~K}$ mixture, and for $r=10$, where an interesting feature in the phase diagram is found regarding the tricritical point [23]. For these mass ratios we present the phase diagrams at three different interaction strengths to see what the effect of the interaction is on the critical temperature. Namely, we present the phase diagrams for a strongly interacting Fermi gas with $1 / k_{F} a=0$, an intermediate interaction strength with $1 / k_{F} a=-1$, and a weakly interacting Fermi gas. These interaction strengths correspond to the BCS side of the Feshbach resonance (see Fig. 1).

Figure 7 shows the phase diagrams of the mass-balanced Fermi gas at three interaction strengths. In Fig. 7(a) the phase diagram for the strongly interacting regime where $1 / k_{F} a=0$ is depicted. It is symmetric in the polarizations. The phase transition is of second order for small polarizations. Moreover, we find two tricritical points in the phase diagram, and below the tricritical points the phase transition is of first order. At a first-order phase transition the order parameter is discontinuous and, therefore, also the particle densities $n_{\sigma}$ [see Eq. (18)]. And thus also the polarization is discontinuous at a first-order phase transition, which gives rise to a forbidden region where phase separation occurs. Figure 7(b) again shows the phase diagram of the mass-balanced Fermi gas, but now for a weaker interaction, namely, for $1 / k_{F} a=-1$. Compared to the unitarity regime, there are no qualitative changes in the phase diagram. However, the critical temperatures are lower than in the strongly interacting regime. Then Fig. 7(c) shows the phase diagram of the mass-balanced Fermi gas for a very weak interaction, namely, $1 / k_{F} a=-3$. Apart from the fact that the critical temperatures are now extremely low, there is a large difference compared to the strongly interacting regime; namely, we do not find tricritical points in the phase diagram, but instead we find two Lifshitz points. Below the Lifshitz point there is an instability toward supersolidity. We assumed that there will be a second-order transition from the normal state to the supersolid. The critical temperature for this transition is found by solving $\alpha\left(\mathbf{k}_{\mathrm{LO}}\right)=0$, where $\mathbf{k}_{\mathrm{LO}}$ is the wavevector of the supersolid. The transition from supersolidity to the homogeneous superfluid phase is expected to be first order [13,19], and thus a forbidden region will be present in the phase diagram. This simple scenario is sketched in the phase diagram, but it could be that the normal-to-supersolid secondorder phase transition is pre-empted by weak first-order transitions from the normal state to various more complicated supersolid phases $[13,19,20]$. For calculation of the relevant fourth-order diagrams with nonzero external momenta, one needs to make an assumption about the crystal structure of the supersolid phase, to calculate a first-order phase transition to the supersolid phase. This calculation for the stability regions of all possible supersolid phases is beyond the scope of this paper. To emphasize that we did not investigate this region in great detail, we use dashed lines in the phase diagram.

Figure 8 again shows three phase diagrams at different interactions, but now for the mass-imbalanced Fermi gas with mass ratio $r=6.7$, corresponding to the ${ }^{6} \mathrm{Li}-{ }^{40} \mathrm{~K}$ Fermi mixture. In the unitarity regime [see Fig. 8(a)], the phase diagram is no longer symmetric in polarizations and the temperatures are lower in comparison with the mass-balanced case. Furthermore, already in the strongly interacting regime we find a Lifshitz point in the phase diagram for a majority of
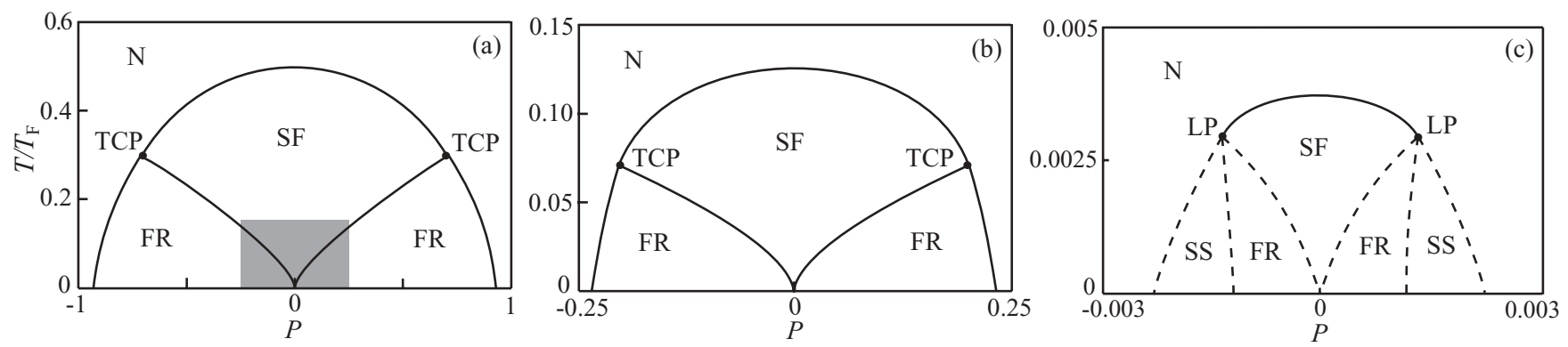

FIG. 7. Phase diagrams of the mass-balanced Fermi gas, $r=1$, as a function of temperature $T$ and polarization $P$ at different interaction strengths. The temperature is scaled with the Fermi temperature $T_{F}$. (a) Phase diagram in the strongly interacting limit, $1 / k_{F} a=0$. There is a tricritical point (TCP), where the normal state $(\mathrm{N})$, the homogeneous superfluid state (SF), and the forbidden region (FR) meet. The shaded area sets the scale for (b), where the phase diagram for a weaker interaction, $1 / k_{F} a=-1$, is shown. Again, there is a TCP. There is again a shaded region to set the scale for (c), but it is too small to see. (c) Phase diagram for a very weak interaction, $1 / k_{F} a=-3$, and the critical temperatures are now extremely low. For this interaction we find a Lifshitz point (LP), below which there is an instability toward a supersolid (SS). The size of this SS region is not calculated within our theory, and therefore the dashed lines are only guides for the eye. 

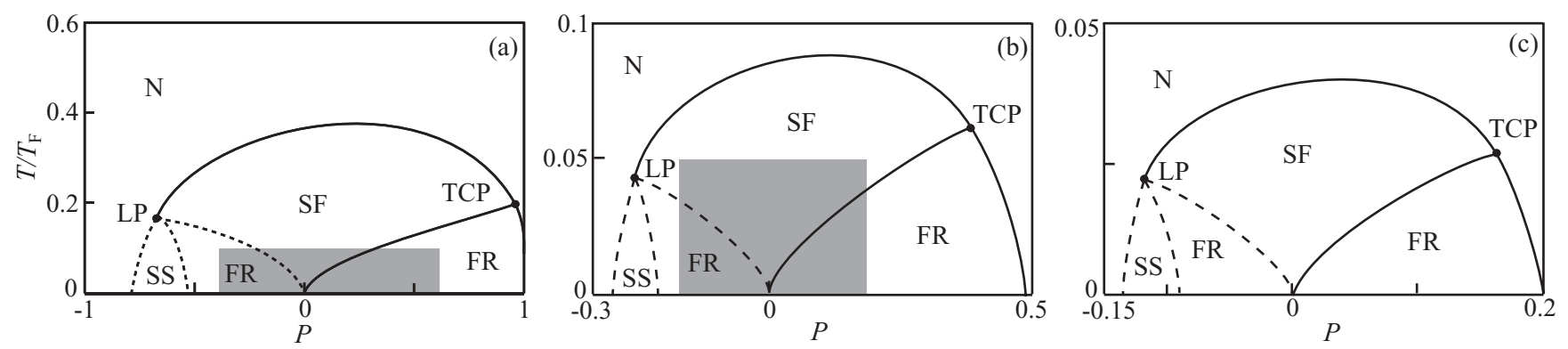

FIG. 8. Phase diagrams of the Fermi gas with mass ratio $r=6.7$ for different interaction strengths. (a) Phase diagram in the strongly interacting limit. The size of the box is the same as for the mass-balanced case, such that the effect of the mass imbalance can be seen. The shaded region sets the scale for (b), where the interaction strength is weaker, $1 / k_{F} a=-1$. (b) The shaded region in turn sets the scale for (c), where the interaction is even weaker, $1 / k_{F} a=-3 / 2$. Dashed lines are guides for the eye.

heavy atoms. For a majority of light particles a tricritical point is found. This is in sharp contrast with the mass-balanced case, where a Lifshitz point is only present for extremely weak interactions. As a result, the Lifshitz temperature is then at least about a hundred times lower than for the ${ }^{6} \mathrm{Li}^{4}{ }^{40} \mathrm{~K}$ mixture. Below the tricritical point there is a forbidden region, and below the Lifshitz point there is an instability toward a supersolid. We sketched the same scenario below the Lifshitz point here as for the mass-balanced case. In Figs. 8(b) and 8(c) the phase diagrams of the Fermi gas are depicted at interaction strengths $1 / k_{\mathrm{F}} a=-1$ and $1 / k_{\mathrm{F}} a=-3 / 2$, respectively. The interaction strength does not affect the topology of the phase diagram, but it does affect the critical temperatures. Just as in the mass-balanced case, the critical temperatures are lower for weaker interactions, as expected.

In Fig. 9 we show the phase diagrams of an imbalanced Fermi gas with an even larger mass imbalance, namely, with mass ratio $r=10$. The diagram has become even more asymmetric. In the strongly interacting regime, we also find a Lifshitz point for a majority of heavy atoms. But the tricritical point has disappeared, which means that the phase transition remains of second order and no phase separation occurs for a majority of light particles. Below the Lifshitz point the same scenario is sketched as before. Figure 9(b) shows the phase diagram at a weaker interaction strength, namely, at $1 / k_{\mathrm{F}} a=-1$. With respect to the unitarity limit there is a real change in this diagram. Namely, the tricritical point reappears in the phase diagram. Then, in Fig. 9(c) the interaction strength is even weaker, $1 / k_{F} a=-3 / 2$, and the critical temperatures are lower. But there are no qualitative changes with respect to the phase diagram in Fig. 9(b).

\section{Effect of a mass imbalance}

By comparing the phase diagrams of the mass-balanced Fermi gas and of the Fermi gases with a mass imbalance, it can be seen that a mass imbalance causes important changes. First, the critical temperatures for the mass-imbalanced Fermi gases are lower than for the mass-balanced case. Second, for a mass-imbalanced Fermi gas the phase diagram is no longer symmetric in polarizations. In other words, the maximum critical temperature is no longer located at zero polarization. Third, for Fermi gases with a sufficiently high mass ratio, like the two mass-imbalanced Fermi gases we present here, there is a Lifshitz point in the phase diagram for a majority of heavy atoms. These three changes we now discuss in more detail.

In the weakly interacting limit, the critical temperatures are very low. For the unpolarized Fermi gas the integral in Eq. (19) can then be evaluated exactly. Then, by equating $\alpha$ to zero, an analytic result for the critical temperature can be obtained [36]:

$$
\begin{aligned}
\left.T_{c}\right|_{P=0} & =\sqrt{\varepsilon_{F,+} \varepsilon_{F,-}} \frac{8}{\pi k_{B}} e^{\gamma-2} e^{-\pi / 2 k_{F}|a|} \\
& =\frac{2 \sqrt{r}}{1+r} \frac{8 \varepsilon_{F}}{\pi k_{B}} e^{\gamma-2} e^{-\pi / 2 k_{F}|a|},
\end{aligned}
$$
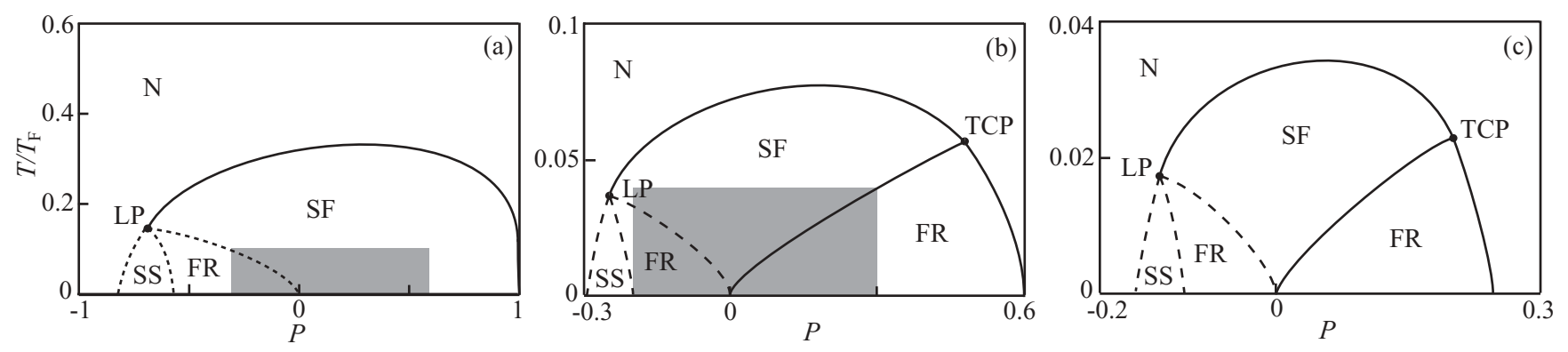

FIG. 9. Phase diagrams of the Fermi gas with mass ratio $r=10$ for different interaction strengths. (a) Phase diagram in the strongly interacting limit. The size of the box is the same as for the mass-balanced case. The shaded region sets the scale for (b), where the interaction strength is weaker, $1 / k_{F} a=-1$. (b) The shaded region in turn sets the scale for (c), where the interaction is even weaker, $1 / k_{F} a=-3 / 2$. Dashed lines are only guides for the eye. 


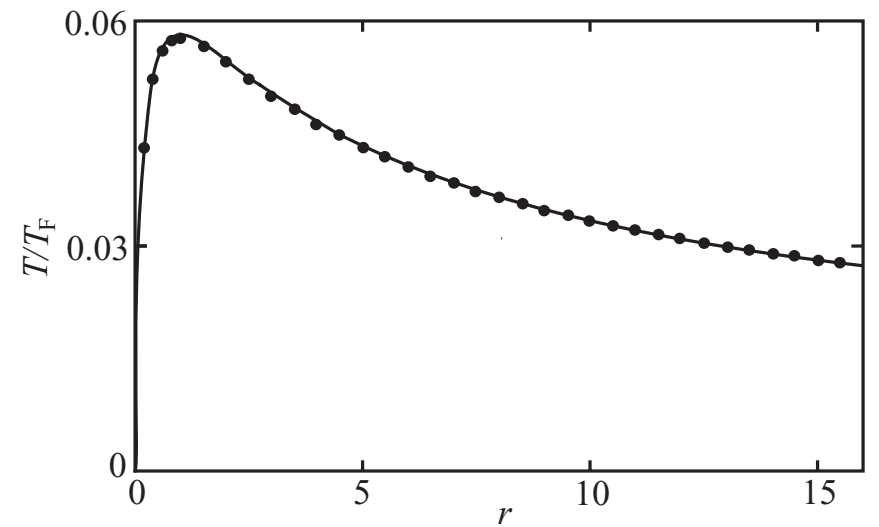

FIG. 10. The critical temperature of an unpolarized Fermi gas as a function of the mass ratio $r=m_{-} / m_{+}$. Circles are the numerical results of $\alpha=0$ and the solid line is the analytic result for the weakly interacting limit. Here $1 / k_{F} a=-3 / 2$.

where $\varepsilon_{F}$ is the Fermi energy corresponding to twice the reduced mass, $k_{B}$ is Boltzmann's constant, and $\gamma=0.5772$ is Euler's constant. When the atoms making up the Fermi mixture have different masses, $r$ is not equal to 1 . In that case the factor $2 \sqrt{r} /(1+r)<1$. And thus, by adding a mass imbalance the critical temperature for the unpolarized Fermi gas becomes lower. Figure 10 shows that the dependence of the critical temperature on the mass ratio is indeed as given by Eq. (26) and that the agreement with the numerical results is very good. For the polarized Fermi gas, we expect a similar dependence of the critical temperature on the mass ratio.

For the mass-balanced Fermi gas, the highest critical temperature is located at zero polarization (see Fig. 7), whereas for the mass-imbalanced Fermi gas it is located at nonzero polarization (see Figs. 8 and 9). As a consequence, the derivative of the critical temperature with respect to $h$, the difference in chemical potentials, will no longer be zero at $P=0$. In the weakly interacting limit, the coefficient $\alpha$ in Eq. (19) can be expanded around the critical temperature $T_{c}$ and the critical "Zeeman" field $h_{0}$ corresponding to the unpolarized Fermi gas,

$$
\begin{aligned}
\alpha\left(T_{c}+\delta T_{c}, h_{0}+\delta h\right) \simeq & \alpha\left(T_{c}, h_{0}\right)+\frac{\partial \alpha}{\partial T}\left(T_{c}, h_{0}\right) \delta T_{c} \\
& +\frac{\partial \alpha}{\partial h}\left(T_{c}, h_{0}\right) \delta h .
\end{aligned}
$$

For a continuous phase transition, $\alpha$ has to go to zero. The term on the left-hand side and the first term on the right-hand side are therefore zero. Then we find, from the other two terms,

$$
\begin{aligned}
\left.\frac{\delta T_{c}}{\delta h}\right|_{P=0} & =-\frac{\partial \alpha / \partial h}{\partial \alpha / \partial T}=\left.T_{c}\right|_{P=0} \frac{1}{4}\left(\frac{1}{\varepsilon_{F,-}}-\frac{1}{\varepsilon_{F,+}}\right) \\
& =\frac{2}{\pi k_{B}} \frac{r-1}{\sqrt{r}} e^{\gamma-2} e^{-\pi / 2 k_{F}|a|} .
\end{aligned}
$$

This is indeed nonzero if the mass ratio is not equal to 1 . Equation (28) is positive for mass ratios $r>1$. This is in agreement with our findings that the phase diagram shifts toward positive polarizations for a mass ratio $r>1$ (see Figs. 8 and 9). For mass ratios $r<1$, Eq. (28) is negative and the phase diagram then shifts toward negative polarizations. In both cases the phase diagram shifts toward a majority of light particles.

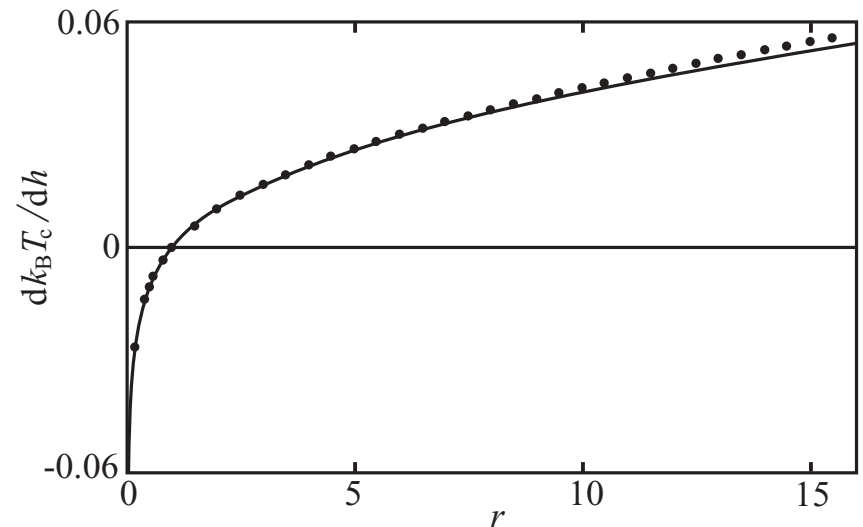

FIG. 11. Derivative of the critical temperature with respect to the difference in chemical potentials $h$. Circles are the numerical results and the solid line is the analytic result. The interaction strength is $1 / k_{F} a=-3 / 2$.

Equation (28) is plotted in Fig. 11, together with the numerical results. The agreement is good, especially for low mass ratios.

For a sufficiently high mass ratio we find a Lifshitz point in the phase diagram for a majority of heavy particles. To explain why this can be expected we consider the kinetic energies of the particles in Eq. (14), which are plotted in Fig. 12. In the mass-balanced case a fermion of one species with momentum $\mathbf{k}$ has the same energy as a fermion with momentum $-\mathbf{k}$ of the other species. Coupling these degenerate states by a condensate of Cooper pairs is energetically desirable since the shift of the energy levels due to the coupling is now largest, as is well known from the physics of avoided crossings. Therefore, it is most favorable to form a pair of two fermions with the same but opposite momentum, that is, a fermion with momentum $\mathbf{k}$ forms a pair with a fermion with momentum $-\mathbf{k}$, since then the energy gain as a result of pairing is maximal. The pair then has no kinetic energy and the pairs form a homogeneous superfluid.

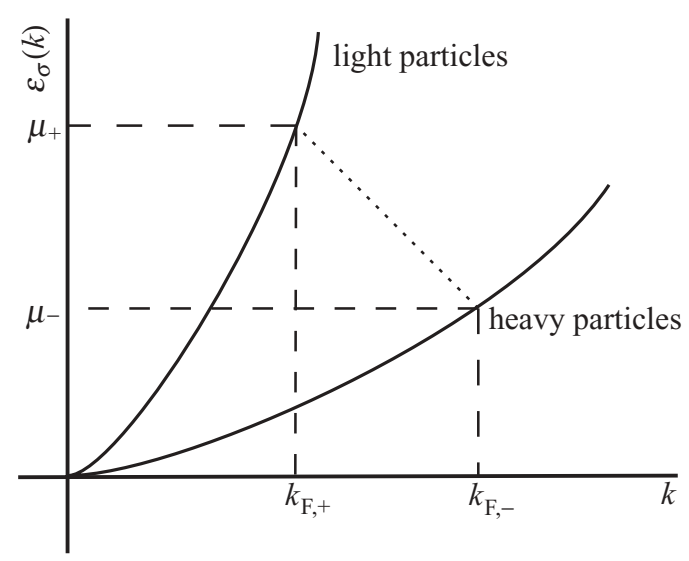

FIG. 12. Energies $\varepsilon_{\sigma}(k)$ of the fermions for a Fermi mixture with both a population and a mass imbalance, where $m_{-}>m_{+}$and $n_{-}>$ $n_{+}$. Thus, there is a majority of heavy particles. At the Fermi levels, the energy difference between particles with different momenta (dashed line) is smaller than the energy difference between particles with the same momenta. 
Typically, the formation of pairs mostly occurs at the Fermi energy. There a pair consists of one fermion with momentum $\mathbf{k}_{\mathrm{F}}$ and one with momentum $-\mathbf{k}_{\mathrm{F}}$. Since the Fermi momentum depends on the density of the particles $n_{\sigma}$ [see Eq. (24)], the Fermi momenta will not be the same in the case of a population imbalance. This makes pairing less ideal than in the unpolarized case. The critical temperatures are therefore lower for a polarized mixture (see Fig. 7).

In the mass-imbalanced case two fermions with equal momentum but from different species do not have the same kinetic energy. Thus, if now a fermion of one species with momentum $\mathbf{k}$ forms a pair with a fermion with momentum $-\mathbf{k}$ of the other species, there is an energy difference that reduces the energy gain that can be obtained due to pairing. This explains the fact that the critical temperatures are lower for Fermi mixtures with a mass imbalance. With both a mass imbalance and a population imbalance, pairs can still be formed with two fermions with equal but opposite momentum, even though there is an energy difference between the Fermi levels. However, this occurs only if the mass ratio and the population imbalance are not too large. In the extreme case of a large mass imbalance and a large population imbalance, a more favorable scenario is possible (see Fig. 12). In Fig. 12 the kinetic energies are plotted for the different fermions, and there is a majority of heavy particles, thus $k_{F,-}>k_{F,+}$. It can be seen that the energy difference between particles with different momenta is now smaller than the energy difference between fermions with the same momentum; that is, it is now energetically more favorable to form pairs with a nonzero momentum that allows for a direct coupling of the Fermi levels. The first point where this is the case is the Lifshitz point. There the system becomes a supersolid.

\section{Multicritical point}

In the phase diagrams, Figs. 7-9, we find, depending on the mass ratio and the interaction strength, tricritical points $\left(P_{\mathrm{c} 3}, T_{\mathrm{c} 3}\right)$, which are solutions of Eq. (11), as well as Lifshitz points $\left(P_{L}, T_{L}\right)$, solutions of Eq. (12).

At unitarity, we find a tricritical point for the mass-balanced case at unitarity, whereas we find a Lifshitz point for the ${ }^{6} \mathrm{Li}^{40} \mathrm{~K}$ mixture for a majority of heavy particles. This Lifshitz point is a clearly distinct point from a tricritical point, just as the tricritical point for the mass-balanced case is a clearly

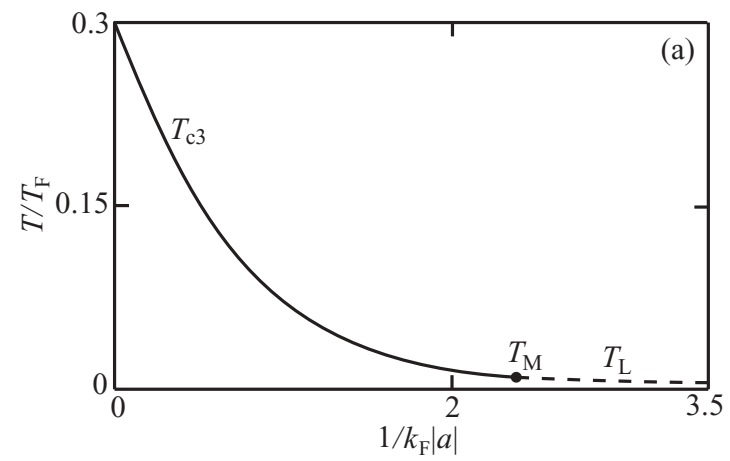

distinct point from a Lifshitz point. This is in accordance with the findings of Parish et al. [37], who found that for a mass-balanced Fermi mixture the FFLO line detaches from the tricritical point away from the BCS limit. However, there is a mass ratio between $r=1$ and $r=6.7$ for which the phase diagram at unitarity contains a point which is both a tricritical point and a Lifshitz point. In other words, for this mass ratio Eqs. (11) and (12) have a solution exactly at the same point in the phase diagram. This is a multicritical point and it is found to occur for the mass ratio $r=4.22$.

It can also be seen in the different phase diagrams that, for a given mass ratio, the location of tricritical and Lifshitz points changes as the interaction strength changes. In Fig. 13, it is shown, for the mass-balanced Fermi gas and for the Fermi gas with mass ratio $r=6.7$ how the temperature $T$ of these points changes as a function of the interaction strength. Note that the polarization is not constant for the lines in Fig. 13. In both Figs. 13(a) and 13(b) one can recognize an exponential decay as a function of the interaction strength, which is exactly what one would expect from Eq. (26).

Figure 13(a) shows the mass-balanced case. In the unitarity regime and for small values of $1 / k_{F}|a|$ a tricritical point is present in the phase diagram, with temperature $T_{\mathrm{c} 3}$. This is the solid line in Fig. 13(a). Then for some value of the interaction strength, Eqs. (11) and (12) have a solution at the same point. Thus, there is a multicritical point and the temperature of this point is denoted $T_{M}$ in Fig. 13. For even weaker interactions it then turns out that there is an instability toward a supersolid and thus we find a Lifshitz point in the phase diagram, with temperature $T_{L}$. This is the dashed line in Fig. 13(a).

Although the tricritical and Lifshitz points are very close together for very weak interactions, in our approach, where we perform the momentum integrals to calculate the coefficients $\gamma$ in Eq. (23) and $\beta$ in Eq. (20), they are essentially always two distinct points, except for one value of the interaction strength where we find a multicritical point. If one, however, assumes particle-hole symmetry in the weakly interacting limit, Eqs. (20) and (23) become, up to a constant, the same equation, and thus one finds that the tricritical point and the Lifshitz point are the same point $[13,38]$.

Figure 13(b) gives the temperature of the Lifshitz point present in the phase diagram of the Fermi gas with mass ratio $r=6.7$ for a majority of heavy particles. Here, we do not

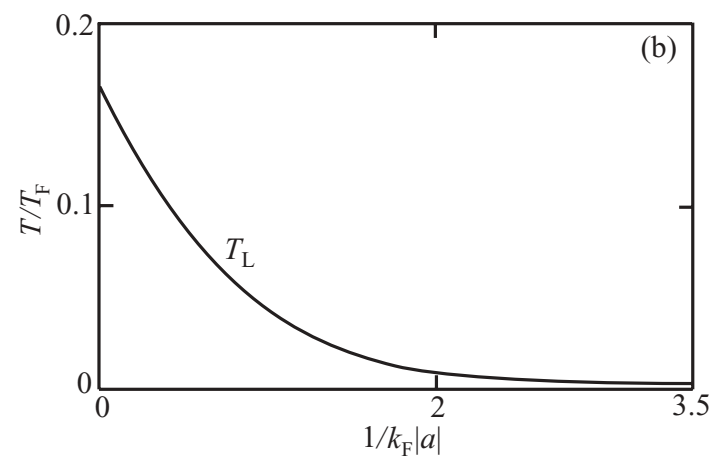

FIG. 13. Temperature of the tricritical points and the Lifshitz points as a function of the interaction strength. (a) The mass-balanced case. (b) A Fermi gas with mass ratio $r=6.7$ and a majority of heavy particles. $T_{\mathrm{c} 3}$ is the temperature of a tricritical point; $T_{M}$, that of a multicritical point; and $T_{L}$, that of a Lifshitz point. 
find a change in character of the phase transition as a function of interaction strength. The Lifshitz point remains a Lifshitz point. For a majority of light particles of this Fermi mixture, we found a tricritical point in the strongly interacting limit. We expect that this point will also change to a Lifshitz point for very weak interactions, but the extremely low temperatures make it numerically difficult to investigate this possibility in detail.

\section{Sarma phase}

In Sec. IV A, in explaining the dispersions of the quasiparticles, we discuss the possibility of a Sarma phase, which occurs when one of the dispersions $\hbar \omega_{\sigma}(\mathbf{k})$ becomes negative. At each point in the phase diagram the value of the order parameter $|\Delta|$ can be found by minimizing the thermodynamic potential in Eq. (13). With this value of the order parameter it can be determined whether the dispersions are always positive or become negative for some range of momenta. In the superfluid phase it turns out that there are different regions. In one region the dispersions are always positive and the superfluid is gapped, while in the other region one of the dispersions becomes negative and we are dealing with a gapless superfluid. At nonzero temperatures, there is no phase transition between these regions. Rather, there is only a smooth crossover. We calculated the gapless superfluid region in the unitarity limit for the mass-balanced case and for the ${ }^{6} \mathrm{Li}^{40} \mathrm{~K}$ Fermi mixture. The results are shown in Fig. 14. In the mass-balanced case, Fig. 14(a), there are two regions in the phase diagram where we have a gapless superfluid. In Fig. 14(b) the mass-imbalanced case is shown. Here, the Sarma region is not symmetric in polarizations, just as the rest of the phase diagram. The Sarma region is very large for positive polarizations, whereas for negative polarizations it becomes very small.

Figure 15 depicts the distribution functions $N_{\sigma}^{\mathrm{SF}}(\mathbf{k})$ of the heavy and the light particles for some point in the phase diagram in Fig. 14(b) that lies in the superfluid Sarma region. In the superfluid phase the distribution functions are modified with respect to the Fermi distribution functions $N_{\sigma}(\mathbf{k})$ and are
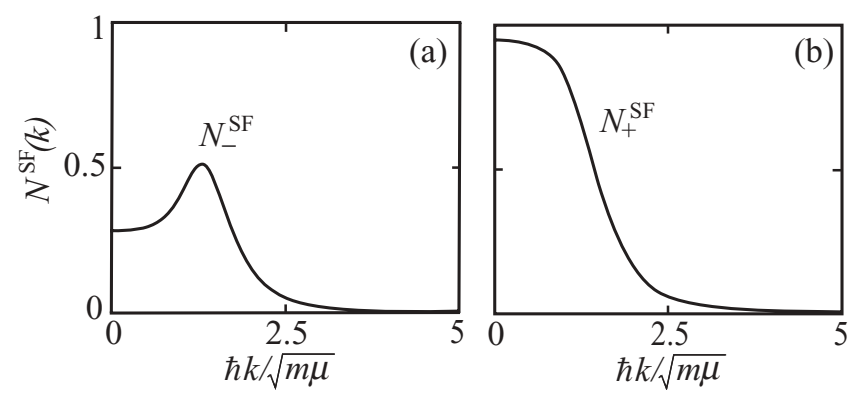

FIG. 15. Distribution functions $N_{\sigma}^{\mathrm{SF}}(\mathbf{k})$ for heavy particles (a) and light particles (b) in the gapless superfluid phase.

given by

$$
\begin{aligned}
N_{\sigma}^{\mathrm{SF}}(\mathbf{k})= & \frac{1}{2}\left[1+\frac{\varepsilon(\mathbf{k})-\mu}{\hbar \omega(\mathbf{k})}\right] N_{\sigma}(\mathbf{k}) \\
& +\frac{1}{2}\left[1-\frac{\varepsilon(\mathbf{k})-\mu}{\hbar \omega(\mathbf{k})}\right]\left[1-N_{-\sigma}(\mathbf{k})\right] .
\end{aligned}
$$

where moreover the quasiparticle dispersions $\hbar \omega_{\sigma}(\mathbf{k})$ are used in $N_{\sigma}(\mathbf{k})$ instead of the normal state dispersions. Figure 15 shows that the distribution function of the heavy particles is nonmonotic, which is a signature of the Sarma phase.

\section{FLUCTUATION EFFECTS}

As argued before, mean-field theory only leads to a qualitative description of the phase transitions that occur in an imbalanced Fermi gas with unitarity-limited interactions. To achieve a quantitative description as well, we have to take fluctuation effects into account. From renormalization-group calculations [25], we know that, especially, self-energy effects and the screening of the interaction by particle-hole fluctuations are important in the unitarity limit. The corresponding corrections result in quantitative agreement with experiments in the mass-balanced case [25] and give rise to more accurate predictions for upcoming experiments with the very promising ${ }^{6} \mathrm{Li}^{-}{ }^{40} \mathrm{~K}$ mixture [14].
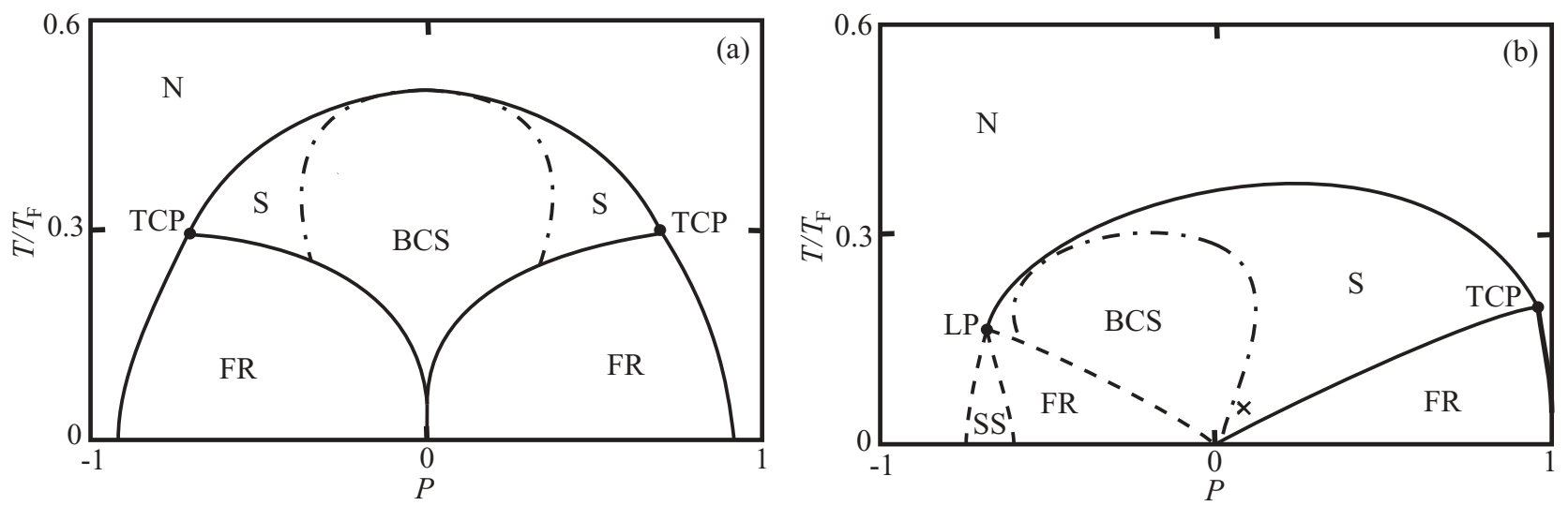

FIG. 14. Phase diagrams at unitarity including the region where the superfluid is gapless. (a) The mass-balanced case. It is the same phase diagram as in Fig. 8(a), only here the superfluid region is divided into a gapped superfluid region (BCS) and two Sarma regions (S). (b) The mass-imbalanced Fermi gas with mass ratio $r=6.7$. The small cross in (b) is the point at which we calculated the distribution functions of the particles shown in Fig. 15. 
(a)

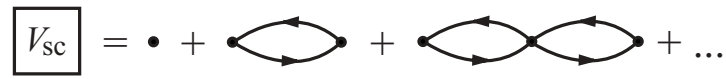

(b)

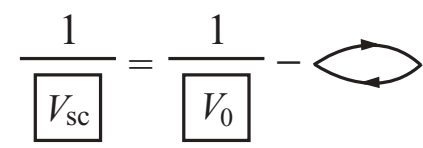

FIG. 16. Screened interaction containing an infinite sum of bubble diagrams. (a) The perturbative expansion of the screened interaction; (b) the result of the resummation of this expansion.

As explained in Refs. [11] and [14], a convenient way to take self-energy corrections into account is to introduce renormalized chemical potentials $\mu_{\sigma}^{\prime}$ that describe the selfenergy of particles with spin $\sigma$ in a Fermi sea of particles with spin $-\sigma$. This can be achieved by using

$$
\mu_{\sigma}^{\prime}=\mu_{\sigma}+c_{\sigma} \frac{\mu_{-\sigma}^{\prime 2}}{\mu_{\sigma}^{\prime}+\mu_{-\sigma}^{\prime}},
$$

where $c_{\sigma}$ is a coefficient that can be determined in the ladder approximation [39], but also with the use of renormalizationgroup calculations [25] or Monte Carlo calculations [41]. For the mass-balanced case, we use $c_{ \pm}=0.6$, while for the ${ }^{6} \mathrm{Li}^{40} \mathrm{~K}$ mixture we use $c_{+}=2.3$ and $c_{-}=0.36$ to incorporate the Monte Carlo results. The substitution of these renormalized chemical potentials $\mu_{\sigma}^{\prime}\left(\mu_{\sigma}\right)$ in the mean-field thermodynamic potential $\omega_{L}\left(\Delta ; \mu_{\sigma}^{\prime}\right)$ results in the following equation for the densities:

$$
n_{\sigma}=-\left.\frac{\partial \omega_{L}\left(\Delta ; \mu_{\sigma}^{\prime}\right)}{\partial \mu_{\sigma}}\right|_{\Delta=\langle\Delta\rangle} .
$$

In Ref. [14], an accurate comparison between the use of renormalized chemical potentials and the use of Monte Carlo calculations was made at zero temperatures, leading to excellent agreement. Note that in the superfluid state, Eq. (30) overestimates the effects of the interactions on the renormalization of the chemical potentials and another correction proportional to $\Delta^{2}$ has to be subtracted on the right-hand side of Eq. (30) [11].

Fluctuations do not affect just the self-energies of the fermions in the normal state. There is another effect of particle-hole fluctuations that affects the transition to the superfluid state. Namely, there is a change in the coefficient $\alpha$ due to screening of the interspecies interaction, which is also called the Gor'kov correction. We can take the screening into account by considering an effective two-body interaction that includes the so-called random-phase approximation (RPA) bubble sum. This procedure is diagrammatically represented in Fig. 16. The inclusion of the infinite geometric series of bubble diagrams leads, at 0 external momentum and frequency, to

$$
\frac{1}{V_{\mathrm{sc}}}=\frac{1}{V_{0}}-\hbar \Pi(\mathbf{0}, 0),
$$

where $\hbar \Pi(\mathbf{0}, 0)$ is the amplitude of the bubble diagram. In Appendix B, it is shown that this amplitude is given by

$$
\hbar \Pi(\mathbf{0}, 0)=\int \frac{d \mathbf{k}^{\prime}}{(2 \pi)^{3}} \frac{N_{+}\left(\mathbf{k}^{\prime}\right)-N_{-}\left(\mathbf{k}^{\prime}\right)}{2 h^{\prime}-\epsilon_{+}\left(\mathbf{k}^{\prime}\right)+\epsilon_{-}\left(\mathbf{k}^{\prime}\right)},
$$

where we use renormalized chemical potentials to also include the fermionic self-energy effects. Using the screened interaction of Eq. (32) in Eq. (4), we obtain a screened two-body transition matrix $T_{\mathrm{sc}}^{2 \mathrm{~B}}(0)$, which consequently enters the expression for the quadratic coefficient in Eq. (19). When the quadratic coefficient including screening becomes zero, a second-order transition can occur, so that the critical condition now becomes

$$
\alpha_{\mathrm{sc}}\left(T_{\mathrm{c}}, \mu_{\sigma}^{\prime}\right)=\alpha\left(T_{\mathrm{c}}, \mu_{\sigma}^{\prime}\right)+\hbar \Pi(\mathbf{0}, 0)=0 .
$$

The new critical condition, which includes both screening and fermionic self-energy effects, typically reduces the obtained critical temperatures by a factor of 3 .

If we apply this procedure to determine the line of secondorder phase transitions in the mass-balanced case, we obtain the result in Fig. 17(a). In this figure, the data along the phase boundaries from the experiment by Shin et al. [9] are also shown. For the unpolarized Fermi gas, we find $T_{\mathrm{c}}=0.18 T_{F}$ and $\mu\left(T_{\mathrm{c}}\right)=0.51 T_{F}$ [14], which is to be compared with the
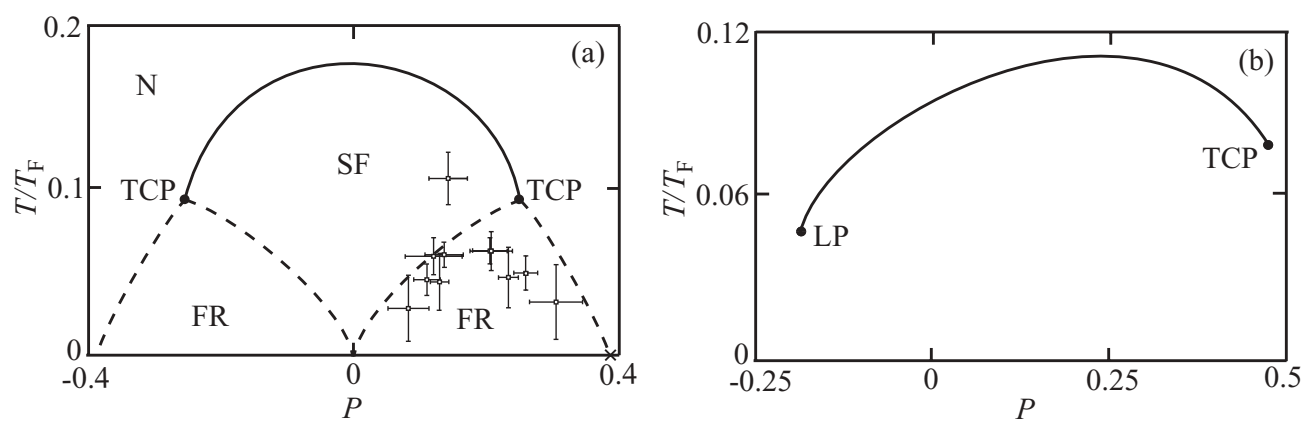

FIG. 17. Phase diagrams of strongly interacting Fermi gases, i.e., $1 / k_{F} a=0$, where fluctuation effects have been taken into account. (a) The mass-balanced case, i.e., $r=1$. Open squares are data along the phase boundaries from the experiment by Shin et al. [9]. Dashed lines denote first-order phase transitions; they were not calculated within our theory and, therefore, are only guides for the eye. However, they include the correct critical polarization at zero temperature, denoted by the cross, known from Monte Carlo calculations [40]. To calculate the first-order phase transition lines more accurately with fluctuation effects taken into account, other methods are needed [11]. (b) Phase diagram for the ${ }^{6} \mathrm{Li}-{ }^{40} \mathrm{~K}$ mixture, where $r=6.7$. For both mixtures the critical temperatures are lowered by taking fluctuation effects into account; compare Figs. 7(a) and 8(a). 
Monte Carlo results $T_{\mathrm{c}}=0.15 T_{F}$ and $\mu\left(T_{\mathrm{c}}\right)=0.49 T_{F}$ [26]. Moreover, for the location of the mass-balanced tricritical point we find $k_{B} T_{\mathrm{c} 3}=0.09 \varepsilon_{F+}$ and $P_{\mathrm{c} 3}=0.24$ [14], which is rather close to the experimental data [9]. We included the critical polarization at zero temperature known from Monte Carlo calculations [40], to be able to sketch the first-order line and the forbidden region. Other methods are needed to calculate the first-order phase transition more accurately with fluctuation effects taken into account [11]. In Fig. 17(b), we show the line of second-order phase transitions for the ${ }^{6} \mathrm{Li}^{40}{ }^{4}$ mixture when fluctuation effects are included. Compared to the mean-field result in Fig. 14(b), the critical temperatures are significantly lower. For the positions of the tricritical point and the Lifshitz point, we use Eqs. (20) and (23), where we again insert the renormalized chemical potentials to include the fermionic selfenergy effects. We then find $k_{B} T_{\mathrm{c} 3}=0.08 T_{F}$ and $P_{\mathrm{c} 3}=0.47$, and $k_{B} T_{L}=0.05 T_{F}$ and $P_{L}=-0.18$, respectively [14]. It is important to note that although the fluctuations have a very large effect quantitatively, the topology of the phase diagrams remains the same.

\section{DISCUSSION AND CONCLUSION}

In this paper we considered Fermi mixtures consisting of two different species of fermions, which can both have a population and a mass imbalance. On the mean-field level we calculated the phase diagrams for those Fermi mixtures as a function of temperature and polarization. We calculated the phase diagrams for different mass ratios and for different interaction strengths, where we found that a mass imbalance leads to a phase diagram that is asymmetrical in the polarization. We also considered the possibility of a Lifshitz instability. We found such instabilities in the Fermi mixtures with a mass ratio of $r=6.7$ and $r=10$ in the unitarity limit. For a mass-balanced mixture, Lifshitz points occur only in the weakly interacting regime.

By studying the effects of a mass imbalance in more detail we found analytic results for the critical temperature and the change in critical temperature at zero polarization. Furthermore, we investigated what happens to the position of the Lifshitz points and the tricritical points when the interaction strength is changed. In the mass-balanced case, we also found a multicritical point for weak interactions. Both for the mass-balanced and for the ${ }^{6} \mathrm{Li}^{40} \mathrm{~K}$ Fermi mixtures, we calculated the regions where the superfluid phase is gapless in the unitarity limit, that is, where the mixtures are in the Sarma phase. These regions were present at nonzero temperatures and turned out to be quite large.

Finally, to obtain more quantitative results we introduced renormalized chemical potentials that include self-energy effects to account for the resonant interactions. We also took screening effects on the critical temperature into account. In this way, we obtained a phase diagram for the mass-balanced mixture that agrees well with Monte Carlo calculations and with experiment. And we hope to have obtained a good quantitative description of the phase diagram for the ${ }^{6} \mathrm{Li}^{40} \mathrm{~K}$ mixture, where the presence of a Lifshitz point is especially exciting. Below the Lifshitz point various supersolid states are competitive. This leads to open questions for further research on the interesting possibility of an inhomogeneous superfluid.

\section{ACKNOWLEDGMENTS}

This work was supported by the Stichting voor Fundamenteel Onderzoek der Materie (FOM) and the Nederlandse Organisatie voor Wetenschaplijk Onderzoek (NWO).

\section{APPENDIX A: DERIVATION OF THE MEAN-FIELD THERMODYNAMIC POTENTIAL}

In this Appendix we explicitly derive the mean-field thermodynamic potential in Eq. (13). We start with the microscopic action for an interacting Fermi mixture consisting of fermions present in two hyperfine states. It is given by

$$
\begin{aligned}
S\left[\phi^{*}, \phi\right]= & \int_{0}^{\hbar \beta} d \tau \int d \mathbf{x} \\
& \times\left\{\sum_{\sigma= \pm} \phi_{\sigma}^{*}(\mathbf{x}, \tau)\left(\hbar \frac{\partial}{\partial \tau}-\frac{\hbar^{2} \nabla^{2}}{2 m_{\sigma}}-\mu_{\sigma}\right) \phi_{\sigma}(\mathbf{x}, \tau)\right. \\
& \left.+V_{0} \phi_{+}^{*}(\mathbf{x}, \tau) \phi_{-}^{*}(\mathbf{x}, \tau) \phi_{-}(\mathbf{x}, \tau) \phi_{+}(\mathbf{x}, \tau)\right\}, \quad(\mathrm{A} 1)
\end{aligned}
$$

where $V_{0} \delta\left(\mathbf{x}-\mathbf{x}^{\prime}\right)$ is the bare pointlike interaction associated with the short-range atomic potentials. The last term in this action is a fourth-order term in the fermionic fields. Therefore, the integral over the fermionic fields in the partition function,

$$
Z=\int d\left[\phi^{*}\right] d[\phi] \exp \left\{-S\left[\phi^{*}, \phi\right] / \hbar\right\},
$$

cannot be performed analytically. To deal with this fourthorder term we introduce, by means of a Hubbard-Stratonovich transformation, bosonic pairing fields $\Delta(\mathbf{x}, \tau)$, which are on average related to the fermionic fields $\phi_{\sigma}(\mathbf{x}, \tau)$ as in Eq. (16). The Hubbard-Stratonovich transformation is performed by inserting into the partition function the following identity:

$$
1=\int d\left[\Delta^{*}\right] d[\Delta] e^{\left(\Delta-\phi_{+} \phi_{-} V_{0}\left|V_{0}^{-1}\right| \Delta^{*}-V_{0} \phi_{-}^{*} \phi_{+}^{*}\right) / \hbar},
$$

where the inner product in the exponent is a short-hand notation for

$$
\begin{gathered}
\int_{0}^{\hbar \beta} d \tau \int d \mathbf{x}\left[\Delta^{*}(\mathbf{x}, \tau)-\phi_{+}^{*}(\mathbf{x}, \tau) \phi_{-}^{*}(\mathbf{x}, \tau) V_{0}\right] \\
\quad \times V_{0}^{-1}\left[\Delta(\mathbf{x}, \tau)-V_{0} \phi_{-}(\mathbf{x}, \tau) \phi_{+}(\mathbf{x}, \tau)\right] .
\end{gathered}
$$

Inserting Eq. (A3) into Eq. (A2) leaves us with an action $S\left[\Delta^{*}, \Delta, \phi^{*}, \phi\right]$, which depends only quadratically on the fermionic fields $\phi_{\sigma}(\mathbf{x}, \tau)$. It is given by

$$
\begin{aligned}
& S\left[\Delta^{*}, \Delta, \phi^{*}, \phi\right] \\
&= \int_{0}^{\hbar \beta} d \tau \int d \mathbf{x}\left\{-\frac{|\Delta(\mathbf{x}, \tau)|^{2}}{V_{0}}-\hbar \sum_{\sigma= \pm} \int_{0}^{\hbar \beta} d \tau^{\prime}\right. \\
& \times \int d \mathbf{x}^{\prime} \phi_{\sigma}^{*}(\mathbf{x}, \tau) G_{0 ; \sigma}^{-1}\left(\mathbf{x}, \tau ; \mathbf{x}^{\prime}, \tau^{\prime}\right) \phi_{\sigma}\left(\mathbf{x}^{\prime}, \tau^{\prime}\right) \\
&\left.+\phi_{+}^{*}(\mathbf{x}, \tau) \phi_{-}^{*}(\mathbf{x}, \tau) \Delta(\mathbf{x}, \tau)+\Delta^{*}(\mathbf{x}, \tau) \phi_{-}(\mathbf{x}, \tau) \phi_{+}(\mathbf{x}, \tau)\right\} .
\end{aligned}
$$


The noninteracting Green's function is given by

$$
\begin{aligned}
& G_{0 ; \sigma}^{-1}\left(\mathbf{x}, \tau ; \mathbf{x}^{\prime}, \tau^{\prime}\right) \\
& \quad=-\frac{1}{\hbar}\left\{\hbar \frac{\partial}{\partial \tau}-\frac{\hbar^{2} \nabla^{2}}{2 m_{\sigma}}-\mu_{\sigma}\right\} \delta\left(\mathbf{x}-\mathbf{x}^{\prime}\right) \delta\left(\tau-\tau^{\prime}\right) .
\end{aligned}
$$

Note that by performing the Hubbard-Stratonovich transformation, we have introduced the order parameter $\Delta(\mathbf{x}, \tau)$ in an exact manner into the many-body theory. If we now integrate out the fermionic fields, we will be left with an effective action $S^{\text {eff }}\left[\Delta^{*}, \Delta\right]$, which is directly related to the Landau free energy. If we apply mean-field theory we assume that the bosonic fields are position and time independent, that is, $\Delta(\mathbf{x}, \tau)=\Delta$. To integrate out the fermionic fields, we need to diagonalize the action. First, Fourier transforming the foregoing action and then writing it in a more compact way using matrix multiplication yields

$$
\begin{aligned}
S\left[\Delta^{*}, \Delta, \phi^{*}, \phi\right]= & -\hbar \beta V \frac{|\Delta|^{2}}{V_{0}}+\hbar \beta \sum_{\mathbf{k}}\left[\varepsilon_{-}(\mathbf{k})-\mu_{-}\right] \\
- & \hbar \sum_{\mathbf{k}, n}\left[\phi_{n,+}^{*}(\mathbf{k}), \phi_{-n,-}(-\mathbf{k})\right] \mathbf{G}_{\Delta}^{-1}\left(\mathbf{k}, i \omega_{n}\right) \\
& \times\left[\begin{array}{c}
\phi_{n,+}(\mathbf{k}) \\
\phi_{-n,-}^{*}(-\mathbf{k})
\end{array}\right]
\end{aligned}
$$

where $V$ is the volume. In expression (A7)

$$
\begin{aligned}
- & \hbar \mathbf{G}_{\Delta}^{-1}\left(\mathbf{k}, i \omega_{n}\right) \\
& =\left[\begin{array}{cc}
-i \hbar \omega_{n}+\varepsilon_{+}(\mathbf{k})-\mu_{+} & \Delta \\
\Delta^{*} & -\left[i \hbar \omega_{n}+\varepsilon_{-}(\mathbf{k})-\mu_{-}\right]
\end{array}\right],
\end{aligned}
$$

where $\omega_{n}$ are the odd Matsubara frequencies. By writing the action in matrix form, we have interchanged the fermionic fields $\phi_{-}$and $\phi_{-}^{*}$, and thereby we have picked up a constant term, namely, the sum $\Sigma_{\mathbf{k}}\left[\varepsilon_{-}(\mathbf{k})-\mu_{-}\right]$in the first line of Eq. (A7). We can now diagonalize this action by means of a Bogoliubov transformation. This transformation consists of unitarily transforming the atomic fields $\phi_{n, \sigma}(\mathbf{k})$ to the quasiparticle fields $\psi_{n, \sigma}(\mathbf{k})$,

$$
\left[\begin{array}{c}
\psi_{n,+}(\mathbf{k}) \\
\psi_{-n,-}^{*}(-\mathbf{k})
\end{array}\right]=\left[\begin{array}{cc}
u(\mathbf{k}) & -v(\mathbf{k}) \\
v^{*}(\mathbf{k}) & u^{*}(\mathbf{k})
\end{array}\right] \cdot\left[\begin{array}{c}
\phi_{n,+}(\mathbf{k}) \\
\phi_{-n,-}^{*}(-\mathbf{k})
\end{array}\right],
$$

where this transformation is unitary if

$$
|u(\mathbf{k})|^{2}+|v(\mathbf{k})|^{2}=1 .
$$

The coefficients of this transformation can be found by demanding the off-diagonal matrix elements to be zero. The action in terms of the fields $\psi_{n, \sigma}(\mathbf{k})$ then reads

$$
\begin{aligned}
S\left[\Delta^{*}, \Delta, \psi^{*}, \psi\right]= & -\hbar \beta V \frac{|\Delta|^{2}}{V_{0}}+\hbar \beta \sum_{\mathbf{k}}[\varepsilon(\mathbf{k})-\mu-\hbar \omega(\mathbf{k})] \\
& +\sum_{\mathbf{k}, n}\left\{\left[-i \hbar \omega_{n}+\hbar \omega_{+}(\mathbf{k})\right] \psi_{n,+}^{*}(\mathbf{k}) \psi_{n,+}(\mathbf{k})\right. \\
& \left.+\left[-i \hbar \omega_{n}+\hbar \omega_{-}(\mathbf{k})\right] \psi_{n,-}^{*}(\mathbf{k}) \psi_{n,-}(\mathbf{k})\right\},
\end{aligned}
$$

where the extra term $\hbar \omega_{+}(\mathbf{k})$ inside the first sum again comes from interchanging fermionic fields. The mean-field partition function reads, after performing the Hubbard-Stratonovich transformation and the Bogoliubov transformation,

$$
\begin{aligned}
Z & =\int d\left[\psi^{*}\right] d[\psi] \exp \left\{-S\left[\Delta^{*}, \Delta, \psi^{*}, \psi\right] / \hbar\right\} \\
& =\exp \left\{\beta V \frac{|\Delta|^{2}}{V_{0}}+\operatorname{Tr}\left[\log \left(-\mathbf{G}^{-1}\right)\right]\right\} \\
& =\exp \left\{-S^{\operatorname{eff}}\left[\Delta^{*}, \Delta\right] / \hbar\right\},
\end{aligned}
$$

where $\mathbf{G}^{-1}$ is

$$
-\hbar \mathbf{G}^{-1}=-\left(\begin{array}{cc}
i \hbar \omega_{n}-\hbar \omega_{+}(\mathbf{k}) & 0 \\
0 & i \hbar \omega_{n}-\hbar \omega_{-}(\mathbf{k})
\end{array}\right) .
$$

The interaction $V_{0}$ in the effective action can be eliminated in favor of the two-body transition matrix using Eq. (4). It is straightforward to calculate the mean-field thermodynamic potential from the effective action, which we find to be

$$
\begin{aligned}
\omega_{\mathrm{L}}(|\Delta|)= & -\frac{1}{\beta V} \log Z \\
= & -\frac{|\Delta|^{2}}{T^{2 \mathrm{~B}}(0)}+\int \frac{d \mathbf{k}}{(2 \pi)^{3}}\{\varepsilon(\mathbf{k})-\mu-\hbar \omega(\mathbf{k}) \\
& \left.+\frac{|\Delta|^{2}}{2 \varepsilon(\mathbf{k})}-\frac{1}{\beta} \sum_{\sigma= \pm} \log \left(1+e^{-\beta \hbar \omega_{\sigma}(\mathbf{k})}\right)\right\},
\end{aligned}
$$

where, to obtain this thermodynamic potential, the sum over the Matsubara frequencies was performed using the following identity:

$$
\sum_{n} \log \left[\beta\left(-i \hbar \omega_{n}+\varepsilon\right)\right]=\log \left(1+e^{-\beta \varepsilon}\right),
$$

which can be derived using contour integration.

\section{APPENDIX B: FEYNMAN DIAGRAMS}

To determine the critical temperature at which a phase transition will take place, either we can, as mentioned in Sec. II, calculate coefficients from the thermodynamic potential by deriving it with respect to $|\Delta|$ or we can use Feynman diagrams. We present the latter scheme in some detail here.

From the action in Eq. (A5) we can determine the propagator for the fermionic fields and the vertices for the interactions between the fermions and the bosons. Namely, the propagator in momentum space is

$$
G_{0, \sigma}\left(\mathbf{k}, i \omega_{n}\right)=\frac{-\hbar}{-i \hbar \omega_{n}+\left[\varepsilon_{\sigma}(\mathbf{k})-\mu_{\sigma}\right]} .
$$

In the Feynman diagrams in Figs. 5(a) and 5(b), this propagator is represented by a straight line, where the plus (minus) indicates a light (heavy) particle. The interaction vertex is proportional to $\delta\left(\mathbf{k}_{\mathbf{1}}+\mathbf{k}_{\mathbf{2}}+\mathbf{k}_{\mathbf{3}}\right) \delta_{n, n^{\prime}, n^{\prime \prime}}$, where $\mathbf{k}_{i}$ are the momenta and $n$ determine the frequencies of the incoming and outgoing particles. This represents nothing but conservation of momentum and energy. In the diagrams in Figs. 5(a) and 5(b) the vertices are the points where three propagators meet. 
To determine the Lifshitz point we need an expression for the ladder diagram with nonzero external momenta.

$$
\begin{aligned}
\frac{1}{\hbar \beta} \sum_{n} \int \frac{d \mathbf{k}}{(2 \pi)^{3}} G_{0,+}\left(\mathbf{q}-\mathbf{k},-i \omega_{n}\right) G_{0,-}\left(\mathbf{k}, i \omega_{n}\right) \\
=\frac{1}{\hbar \beta} \sum_{n} \int \frac{d \mathbf{k}}{(2 \pi)^{3}} \frac{-\hbar}{i \omega_{n} \hbar+\left[\varepsilon_{+}(\mathbf{q}-\mathbf{k})-\mu_{+}\right]} \\
\quad \times \frac{-\hbar}{-i \omega_{n} \hbar+\left[\varepsilon_{-}(\mathbf{k})-\mu_{-}\right]} .
\end{aligned}
$$

We can split the fractions and then perform the summation over the Matsubara frequencies. This results in

$$
\int \frac{d \mathbf{k}}{(2 \pi)^{3}} \frac{N_{+}(\mathbf{q}-\mathbf{k})+N_{-}(\mathbf{k})-1}{\varepsilon_{+}(\mathbf{q}-\mathbf{k})+\varepsilon_{-}(\mathbf{k})-2 \mu} .
$$

If we want to obtain the full expression for the quadratic part, we have to add the terms proportional to $|\Delta|^{2}$ from the effective action. By doing so we obtain $\alpha(\mathbf{q})$ in Eq. (21). In the same way the diagrams needed for $\alpha$ in Eq. (19) and for $\beta$ in Eq. (20) can be calculated.

When we include fluctuation effects, we also take the screening of the interaction by the particle-hole fluctuations into account. We do so by replacing the bare interaction potential $V_{0}$ with a screened interaction potential $V_{\mathrm{sc}}$ containing the infinite sum of so-called RPA bubble diagrams (see Fig. 16). The sum over all the bubble diagrams in Fig. 16 is a geometric series. Using this, we find the result for the screened interaction, Eq. (32). Thus, to find the screened interaction, we have to calculate the amplitude of the bubble diagram. The bubble diagram consists of two fermionic propagators with momentum and energy going in opposite directions through the diagram. The amplitude is given by

$$
\begin{aligned}
& \frac{1}{\hbar \beta} \sum_{n} \int \frac{d \mathbf{k}}{(2 \pi)^{3}} \frac{-\hbar}{-i \hbar \omega_{n}+\varepsilon_{+}(\mathbf{k})-\mu_{+}} \\
& \quad \times \frac{-\hbar}{-i \hbar \omega_{n}+\varepsilon_{-}(\mathbf{k})-\mu_{-}} .
\end{aligned}
$$

Writing expression (B3) as the sum of two fractions and then performing the sum over the Matsubara frequencies results in

$$
\hbar \Pi(\mathbf{0}, 0)=\int \frac{d \mathbf{k}}{(2 \pi)^{3}} \frac{N_{+}(\mathbf{k})-N_{-}(\mathbf{k})}{2 h-\left[\varepsilon_{+}(\mathbf{k})-\varepsilon_{-}(\mathbf{k})\right]} .
$$

[1] C. A. Regal, M. Greiner, and D. S. Jin, Phys. Rev. Lett. 92, 040403 (2004).

[2] M. W. Zwierlein, C. A. Stan, C. H. Schunck, S. M. F. Raupach, A. J. Kerman, and W. Ketterle, Phys. Rev. Lett. 92, 120403 (2004).

[3] J. Kinast, S. L. Hemmer, M. E. Gehm, A. Turlapov, and J. E. Thomas, Phys. Rev. Lett. 92, 150402 (2004).

[4] M. Bartenstein, A. Altmeyer, S. Riedl, S. Jochim, C. Chin, J. H. Denschlag, and R. Grimm, Phys. Rev. Lett. 92, 203201 (2004).

[5] T. Bourdel, L. Khaykovich, J. Cubizolles, J. Zhang, F. Chevy, M. Teichmann, L. Tarruell, S. J. J. M. F. Kokkelmans, and C. Salomon, Phys. Rev. Lett. 93, 050401 (2004).

[6] G. B. Partridge, K. E. Strecker, R. I. Kamar, M. W. Jack, and R. G. Hulet, Phys. Rev. Lett. 95, 020404 (2005).

[7] M. W. Zwierlein et al., Science 311, 492 (2006).

[8] G. B. Partridge et al., Science 311, 503 (2006).

[9] Y. Shin et al., Nature 451, 689 (2008).

[10] K. B. Gubbels, M. W. J. Romans, and H. T. C. Stoof, Phys. Rev. Lett. 97, 210402 (2006).

[11] J. M. Diederix, K. B. Gubbels, and H. T. C. Stoof, e-print arXiv:0907.0127v2.

[12] D. Bailin and A. Love, Phys. Rep. 107, 325 (1984).

[13] R. Casalbuoni and G. Nardulli, Rev. Mod. Phys. 76, 263 (2004).

[14] K. B. Gubbels, J. E. Baarsma, and H. T. C. Stoof, Phys. Rev. Lett. 103, 195301 (2009).

[15] A. I. Larkin and Y. N. Ovchinnikov, Zh. Eksp. Teor. Fiz. 47, 1136 (1964).

[16] P. Fulde and R. A. Ferrell, Phys. Rev. 135, A550 (1964).

[17] A. Bulgac and M. M. Forbes, Phys. Rev. Lett. 101, 215301 (2008).
[18] Y. Liao et al., e-print arXiv:0912.0092.

[19] C. Mora and R. Combescot, Phys. Rev. B 71, 214504 (2005).

[20] N. Yoshida and S.-K. Yip, Phys. Rev. A 75, 063601 (2007).

[21] E. Wille, M. Taglieber, L. Costa, T. Aoki, W. Wieser, T. W. Hansch, and K. Dieckmann, Phys. Rev. Lett. 100, 053201 (2008).

[22] A.-C. Voigt et al., Phys. Rev. Lett. 102, 020405 (2009).

[23] M. M. Parish, F. M. Marchetti, A. Lamacraft, and B. D. Simons, Phys. Rev. Lett. 98, 160402 (2007).

[24] L. P. Gor'kov and T. K. Melik-Barkhudarov, Sov. Phys. JETP 13, 1018 (1961).

[25] K. B. Gubbels and H. T. C. Stoof, Phys. Rev. Lett. 100, 140407 (2008).

[26] E. Burovski, N. Prokofev, B. Svistunov, and M. Troyer, Phys. Rev. Lett. 96, 160402 (2006).

[27] R. A. Duine and H. T. C. Stoof, Phys. Rep. 396, 115 (2004).

[28] M. W. J. Romans and H. T. C. Stoof, Phys. Rev. Lett. 95, 260407 (2005).

[29] J. W. Negele and H. Orland, Quantum Many-Particle Systems (Westview Press, Boulder, CO, 1998).

[30] H. T. C. Stoof, K. B. Gubbels, and D. B. M. Dickerscheid, Ultracold Quantum Fields (Springer, Dordrecht, 2009).

[31] P. M. Chaikin and T. C. Lubensky, Principles of Condensed Matter Physics (Cambridge University Press, Cambridge, 1995).

[32] M. Iskin and C. A. R. Sá de Melo, Phys. Rev. A 77, 013625 (2008).

[33] A. L. Fetter, J. D. Walecka, Quantum Theory of Many-Particle Systems (McGraw-Hill, New York, 1971).

[34] G. Sarma, J. Phys. Chem. Solids 24, 1029 (1963). 
[35] H. Kleinert, Fortschr. Phys. 26, 565 (1978).

[36] T. Paananen, P. Törmä, and J.-P. Martikainen, Phys. Rev. A 75, 023622 (2007).

[37] M. M. Parish et al., Nature Phys. 3, 124 (2007).

[38] A. I. Buzdin and H. Kachkachi, Phys. Lett. A 225, 341 (1997).
[39] R. Combescot, A. Recati, C. Lobo, and F. Chevy, Phys. Rev. Lett. 98, 180402 (2007).

[40] C. Lobo, A. Recati, S. Giorgini, and S. Stringari, Phys. Rev. Lett. 97, 200403 (2006).

[41] A. Gezerlis et al., e-print arXiv:0901.3105. 